Survey Paper

\title{
Three-dimensional solid texture analysis in biomedical imaging: Review and opportunities
}

\author{
Adrien Depeursinge ${ }^{\mathrm{a}, \mathrm{b}, \mathrm{d}, *}$, Antonio Foncubierta-Rodriguez ${ }^{\mathrm{a}}$, Dimitri Van De Ville ${ }^{\mathrm{b}, \mathrm{c}}$, Henning Müller ${ }^{\mathrm{a}, \mathrm{b}}$ \\ ${ }^{a}$ Business Information Systems, University of Applied Sciences Western Switzerland (HES-SO), Sierre, Switzerland \\ ${ }^{\mathrm{b}}$ Department of Radiology, University and University Hospitals of Geneva (HUG), Switzerland \\ ' Ecole Polytechnique, Fédérale de Lausanne (EPFL), Switzerland \\ ${ }^{\mathrm{d}}$ Department of Radiology, School of Medicine, Stanford University, CA, USA
}

\section{A R T I C L E I N F O}

\section{Article history:}

Received 8 January 2013

Received in revised form 10 October 2013

Accepted 10 October 2013

Available online 22 October 2013

\section{Keywords:}

3-D texture

Volumetric texture

Solid texture

Texture primitive

Classification

\begin{abstract}
A B S T R A C T
Three-dimensional computerized characterization of biomedical solid textures is key to large-scale and high-throughput screening of imaging data. Such data increasingly become available in the clinical and research environments with an ever increasing spatial resolution. In this text we exhaustively analyze the state-of-the-art in 3-D biomedical texture analysis to identify the specific needs of the application domains and extract promising trends in image processing algorithms. The geometrical properties of biomedical textures are studied both in their natural space and on digitized lattices. It is found that most of the tissue types have strong multi-scale directional properties, that are well captured by imaging protocols with high resolutions and spherical spatial transfer functions. The information modeled by the various image processing techniques is analyzed and visualized by displaying their 3-D texture primitives. We demonstrate that non-convolutional approaches are expected to provide best results when the size of structures are inferior to five voxels. For larger structures, it is shown that only multi-scale directional convolutional approaches that are non-separable allow for an unbiased modeling of 3-D biomedical textures. With the increase of high-resolution isotropic imaging protocols in clinical routine and research, these models are expected to best leverage the wealth of 3-D biomedical texture analysis in the future. Future research directions and opportunities are proposed to efficiently model personalized image-based phenotypes of normal biomedical tissue and its alterations. The integration of the clinical and genomic context is expected to better explain the intra class variation of healthy biomedical textures. Using texture synthesis, this provides the exciting opportunity to simulate and visualize texture atlases of normal ageing process and disease progression for enhanced treatment planning and clinical care management. (c) 2013 Elsevier B.V. All rights reserved.
\end{abstract}

\section{Introduction}

The mature field of imaging physics brought a large variety of irreplaceable diagnosis and research tools to the clinicians and biologists. These tools are perfectly aligned with evidence-based medicine aiming to take decisions based on proven facts (Simel and Drummond, 2008). Since the 1970s, tomographic imaging devices such as X-ray computed tomography (CT) and magnetic resonance imaging (MRI), 3-D ultrasound (US) since the late 1980s have allowed observing the human body in 3-D with sub-millimetric voxel dimensions. These tools are widely used in clinical routine and research. Research in biology has also been relying extensively on confocal microscopy providing 3-D images with sub-micromet-

\footnotetext{
* Corresponding author at: Business Information Systems, University of Applied Sciences Western Switzerland (HES-SO), Sierre, Switzerland.

E-mail addresses: adrien.depeursinge@hevs.ch, adrien.depeursinge@gmail.com (A. Depeursinge).
}

ric resolution and specific contrast thanks to fluorescent markers (Pawley, 2006). When compared to bi-dimensional images, 3-D volumetric image series cannot be visualized comprehensively and MPR (Multi-Planar Rendering) or semi-transparent rendering is needed to navigate through the various parts of the observed organ. The image interpretation process is time-consuming and error-prone since the radiologists or researchers in biology have to exhaustively browse image series having sometimes several thousand slices (Andriole et al., 2011). As a consequence, computerized analysis of 3-D data has become one of the major research subjects in medical imaging and diagnostic radiology, known as computer-aided diagnosis (CAD) (Duncan and Ayache, 2000; Doi, 2007). The goal of CAD is to use computer vision to assist radiologists in focusing their attention on diagnostically relevant events and to provide quantitative measures for suspicious biomedical tissue, as well as automatic segmentation of anomalies and retrieval of similar cases (Müller et al., 2004; Nishikawa, 2007). Recent imaging devices have been reaching increasingly high spatial 


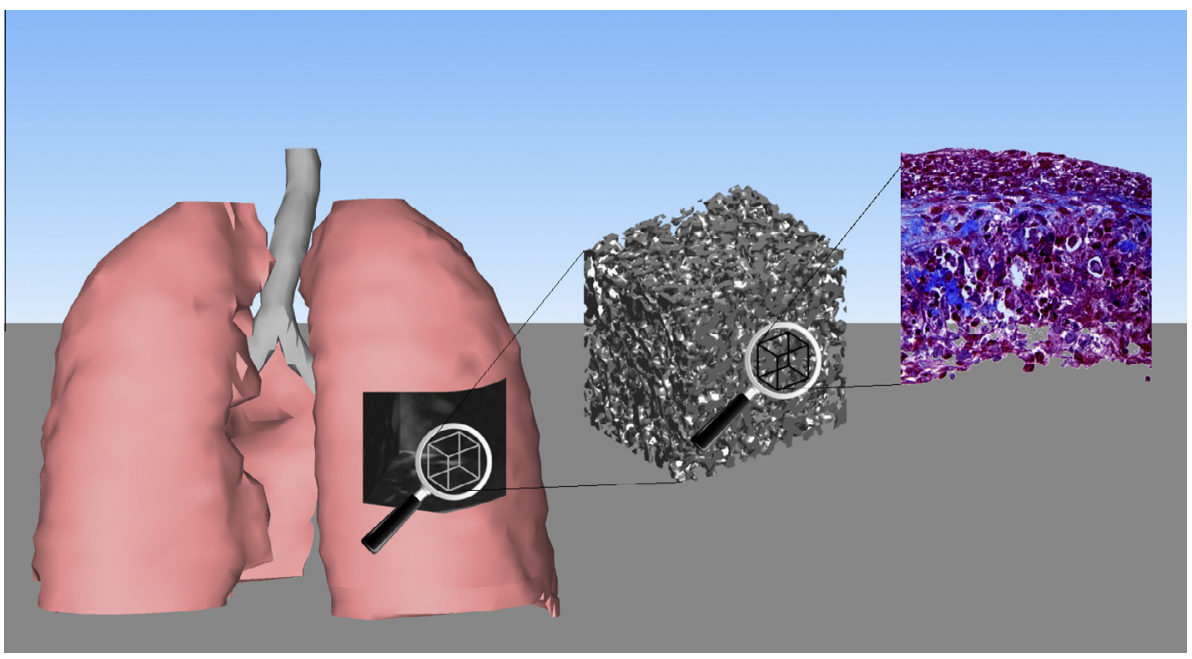

Fig. 1. 3-D biomedical tissue defines solid texture at multiple scales.

resolution, allowing to characterize structural properties of biomedical tissue. ${ }^{1}$ Tissue anomalies are well characterized by localized texture properties in most imaging modalities (Tourassi, 1999; Kovalev and Petrou, 2000; Castellano et al., 2004; Depeursinge and Müller, 2011). This calls for scientific contributions on computerized analysis of 3-D texture in biomedical images, which engendered major scientific breakthroughs in 3-D solid texture analysis during the past 20 years (Blot and Zwiggelaar, 2002; Kovalev and Petrou, 2009; Foncubierta-Rodríguez et al., 2013a).

\subsection{Biomedical volumetric solid texture}

A uniform textured volume in a 3-D biomedical image is considered to be composed of homogeneous tissue properties. The concept of organs or organelles was invented by human observers for efficient understanding of anatomy. The latter can be defined as an organized cluster of one or several tissue types (i.e., defining solid textures). Fig. 1 illustrates that, at various scales, everything is texture in biomedical images starting from the cell level to the organ level. The scaling parameter of textures is thus fundamental and it is often used in computerized texture analysis approaches (Yeshurun and Carrasco, 2000).

According to the Oxford Dictionaries, ${ }^{2}$ texture is defined as "the feel, appearance, or consistency of a surface or a substance", which relates to the surface structure or the internal structure of the considered matter in the context of 2-D or 3-D textures, respectively. The definition of 3-D texture is not equivalent to 2-D surface texture since opaque 3-D textures cannot be described in terms of reflectivity or albedo of a matter, which are often used to characterize textured surfaces (Dana et al., 1999). Haralick et al. (1973) also define texture as being "an innate property of virtually all surfaces" and stipulates that texture "contains important information about the structural arrangement of surfaces and their relationship to the surrounding environment", which is also formally limited to textured surfaces.

Textured surfaces are central to human vision, because they are important visual cues about surface property, scenic depth, surface orientation, and texture information is used in pre-attentive vision for identifying objects and understanding scenes (Julesz, 1962). The human visual cortex is sensitive to the orientation and spatial frequencies (i.e., repetitiveness) of patterns (Blakemore and

\footnotetext{
1 Biomedical tissue is considered in a broad meaning including connective, muscle, and nervous tissue.

2 http://oxforddictionaries.com/definition/texture, as of 9 October 2013.
}

Campbell, 1969; Maffei and Fiorentini, 1973), which relates to texture properties. It is only in a second step that regions of homogeneous textures are aggregated to constitute objects (e.g., organs) at a higher level of scene interpretation. However, the human comprehension of the three-dimensional environment relies on objects. The concept of three-dimensional texture is little used, because texture existing in more than two dimensions cannot be fully visualized by humans (Toriwaki and Yoshida, 2009). Only virtual navigation in MPR or semi-transparent visualizations are made available by computer graphics and allow observing 2-D projections of opaque textures.

In a concern of sparsity and synthesis, 3-D computer graphics have been focusing on objects. Shape-based methods allow encapsulating essential properties of objects and thus provide approximations of the real world that are corresponding to human understanding and abstraction level. Recently, data acquisition techniques in medical imaging (e.g., tomographic, confocal, echographic) as well as recent computing and storage infrastructures allow computer vision and graphics to go beyond shape-based methods and towards three-dimensional solid texture-based description of the visual information. 3-D solid textures encompass rich information of the internal structures of objects because they are defined for each coordinate $x, y, z \in V_{x, y, z} \subset \mathbb{R}^{3}$, whereas shapebased descriptions are defined on surfaces $x, y, z \in \Gamma_{u, v} \subset \mathbb{R}^{3}$. $|V| \gg|\Gamma|$ because every point of $\Gamma$ can be uniquely indexed by only two coordinates $(u, v)$. Texture- and shape-based approaches are complementary and their success depends on the application needs. While several papers on shape-based methods for classification and retrieval of organs and biomedical structures have been published during the past 15 years (McInerney and Terzopoulos, 1996; Metaxas, 1996; Beichel et al., 2001; Heimann and Meinzer, 2009), 3-D biomedical solid texture analysis is still an emerging research field (Blot and Zwiggelaar, 2002). The most common approach to 3-D solid texture analysis is to use 2-D texture in slices (Castellano et al., 2004; Depeursinge et al., 2007; Sørensen et al., 2010) or by projecting volumetric data on a plane (Chan et al., 2008), which does not allow exploiting the wealth of 3-D texture information. Based on the success and attention that 2-D texture analysis obtained in the biomedical computer vision community as well as the observed improved performance of 3-D techniques over 2-D approaches in several application domains (Ranguelova and Quinn, 1999; Mahmoud-Ghoneim et al., 2003; Xu et al., 2006b; Chen et al., 2007), 3-D biomedical solid texture analysis is expected to be a major research field in computer vision in the coming years. The extension of 2-D approaches to $\mathbb{R}^{3}$ (or $\mathbb{Z}^{3}$ for 
sampled lattices) is not straightforward and raises several challenges related to translation, scaling and rotation invariances and covariances that are becoming more complex in 3-D.

\subsection{Related work and scope of this survey}

Depending on research communities, various taxonomies are used to refer to 3-D texture information. A clarification of the taxonomy is proposed in this section to accurately define the scope of this survey. It is partly based on Toriwaki and Yoshida, 2009. Threedimensional texture and volumetric texture are both general and identical terms designing a texture defined in $\mathbb{R}^{3}$ and include:

(1) Volumetric textures existing in "filled" objects $\left\{V: x, y, z \in V_{x, y, z} \subset \mathbb{R}^{3}\right\}$ that are generated by a volumetric data acquisition device (e.g., tomography, confocal imaging).

(2) $2.5 \mathrm{D}$ textures existing on surfaces of "hollow" objects as $\left\{\Gamma: x, y, z \in \Gamma_{u, v} \subset \mathbb{R}^{3}\right\}$,

(3) Dynamic textures in two-dimensional time sequences as $\left\{S: x, y, t \in S_{x, y, t} \subset \mathbb{R}^{3}\right\}$,

Solid texture refers to category (1) and accounts for textures defined in a volume $V_{x, y, z}$ indexed by three coordinates. Solid textures have an intrinsic dimension of 3 , which means that a number of variables equal to the dimensionality of the Euclidean space is needed to represent the signal (Bennett, 1965; Foncubierta-Rodríguez et al., 2013a). Category (2) is designed as textured surface in Dana and Nayar (1999) and Cula and Dana (2004), or 2.5-dimensional textures in Lu et al. (2006) and Aguet et al. (2008), where textures $\Gamma$ are existing on the surface of 3-D objects and can be indexed uniquely by two coordinates $(u, v)$. (2) is also used in Kajiya and Kay (1989), Neyret (1995), and Filip and Haindl (2009), where 3-D geometries are added onto the surface of objects to create realistic rendering of virtual scenes. Motion analysis in videos can also be considered a multi-dimensional texture analysis problem belonging to category (3) and is designed by "dynamic texture" in Bouthemy and Fablet (1998), Chomat and Crowley (1999), and Schödl et al. (2000).

In this survey, a comprehensive review of the literature published on classification and retrieval of biomedical solid textures (i.e., category (1)) is carried out. The focus of this text is on the feature extraction and not machine learning techniques, since only feature extraction is specific to 3-D solid texture analysis.

\subsection{Structure of this article}

This survey is structured as follows: The fundamentals of 3-D digital texture processing are defined in Section 2. Section 3 describes the review methodology used to systematically retrieve papers dealing with 3-D solid texture classification and retrieval. The imaging modalities and organs studied in the literature are reviewed in Sections 4 and 5, respectively to list the various expectations and needs of 3-D image processing. The resulting applicationdriven techniques are described, organized and grouped together in Section 6. A synthesis of the trends and gaps of the various approaches, conclusions and opportunities are given in Section 7 , respectively.

\section{Fundamentals of solid texture processing}

Although several researchers attempted to establish a general model of texture description (Haralick, 1979; Julesz, 1981), it is generally recognized that no general mathematical model of texture can be used to solve every image analysis problem (Mallat, 1999). In this survey, we compare the various approaches based on the 3-D geometrical properties of the primitives used, i.e., the elementary building block considered. The set of primitives used and their assumed interactions define the properties of the texture analysis approaches, from statistical to structural methods.

In Section 2.1, we define the mathematical framework and notations considered to describe the content of 3-D digital images. The notion of texture primitives as well as their scales and directions are defined in Section 2.2.

\subsection{3-D digitized images and sampling}

In Cartesian coordinates, a generic 3-D continuous image is defined by a function of three variables $f(x, y, z)$, where $f$ represents a scalar at a point $(x, y, z) \in \mathbb{R}^{3}$. A 3-D digital image $F(i, j, k)$ of dimensions $M \times N \times O$ is obtained from sampling $f$ at points $(i, j, k) \in \mathbb{Z}^{3}$ of a 3-D ordered array (see Fig. 2). Increments in ( $i, j, k)$, correspond to physical displacements in $\mathbb{R}^{3}$ parametrized by the respective spacings $(\Delta x, \Delta y, \Delta z)$. For every cell of the digitized array, the value of $F(i, j, k)$ is typically obtained by averaging $f$ in the cuboid domain defined by $(x, y, z) \in[i \Delta x,(i+1) \Delta x] ; \quad[j \Delta y,(j+1) \Delta y] ; \quad[k \Delta z,(k+$ 1) $\Delta z]$ ) (Toriwaki and Yoshida, 2009). This cuboid is called a voxel. The three spherical coordinates $(r, \theta, \phi)$ are unevenly sampled to $(R, \Theta, \Phi)$ as shown in Fig. 3.

\subsection{Texture primitives}

The notion of texture primitive has been widely used in 2-D texture analysis and defines the elementary building block of a given texture class (Haralick, 1979; Jain et al., 1995; Lin et al., 1999). All texture processing approaches aim at modeling a given texture using sets of prototype primitives. The concept of texture primitive is naturally extended in 3-D as the geometry of the voxel sequence used by a given texture analysis method. We consider a primitive $\Gamma(i, j, k)$ centered at a point $(i, j, k)$ that lives on a neighborhood of this point. The primitive is constituted by a set of voxels with gray tone values that forms a 3-D structure. Typical $\Gamma$ neighborhoods are voxel pairs, linear, planar, spherical or unconstrained. Signal assignment to the primitive can be either binary, categorical or continuous. Two example texture primitives are shown in Fig. 4. Texture primitives refer to local processing of 3-D images and local patterns (see Toriwaki and Yoshida, 2009).

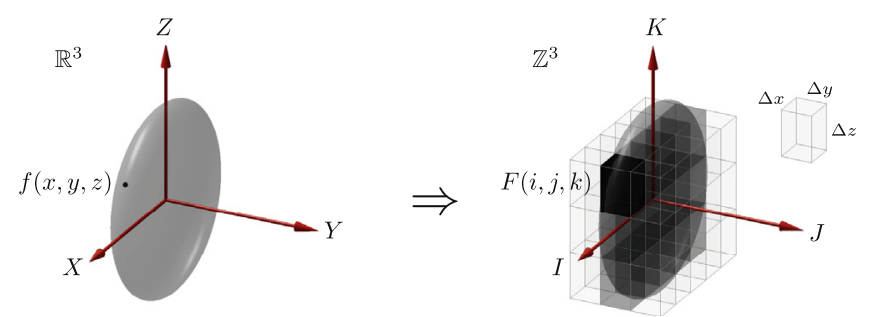

Fig. 2. 3-D digitized images and sampling in Cartesian coordinates.

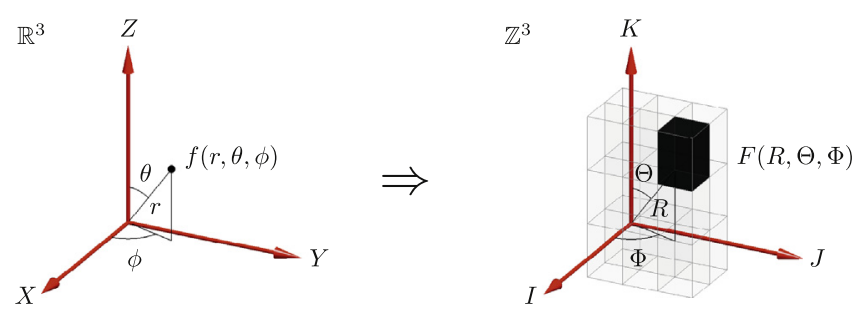

Fig. 3. 3-D digitized images and sampling in spherical coordinates. 

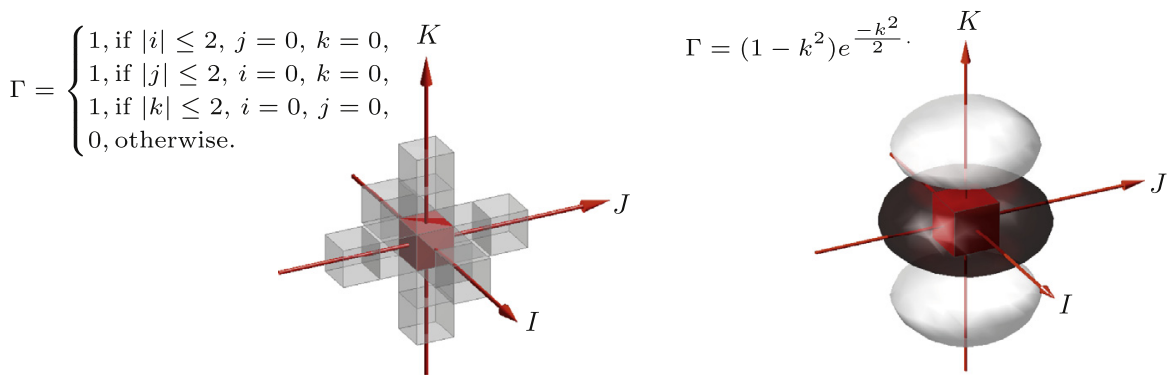

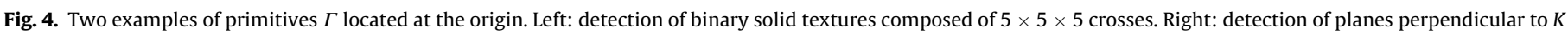
in quantized images.

\subsubsection{Scales and directions}

The most elementary primitive is one isolated voxel, whereas the most comprehensive is the whole image. In between are primitives of support $2 R+1$. The support of the primitive relates to the scale of the texture. The number of possible directions $(\Theta, \Phi)$ directly depends on the scale of the primitive as $(2 R+1)^{3}-1$ (see Fig. 3). The relative position of the primitive and the central voxel relates to the phase of the primitive along a given direction.

\section{Review methodology}

A systematic review methodology was used in order to obtain a clear overview of the state-of-the-art in 3-D biomedical texture analysis. In a first step, five publication search engines from the important societies and publishers in computer science were queried using keywords that are specific to the classification and retrieval of biomedical 3-D textures. The search engines used are SciVerse Scopus, ${ }^{3}$ ACM Digital Library, ${ }^{4}$ PubMed, ${ }^{5}$ IEEE Xplore ${ }^{6}$ and SPIE Digital Library. ${ }^{7}$ After a preliminary analysis of this initial collection of papers, it became clear that SciVerse Scopus was including results from all other search engines as papers from (but not limited to) ACM Digital Library, Elsevier, ${ }^{8}$ IEEE Xplore, SPIE Digital Library and Springer. ${ }^{9}$ All subsequent searches for papers containing query keywords in the abstract, title or keywords were performed exclusively in Scopus as follows:

1. "retrieval" AND “3D": 4005 papers,

- refined with "texture": 230,

- refined with "medical" OR "health": 316 ,

- refined with "CT" OR "MRI" OR "PET": 211,

- refined with "voxel": 78,

- union of the refined results: 689 papers.

2. "CBIR" AND “3D": 35

- union with (1): 703 papers.

3. "texture" AND “classification" AND "3D": 375 ,

- union with (2): 1033 papers.

4. ("medical" OR "health") AND "classification" AND “3D": 640,

- union with (3): 1589 papers.

This initial set of 1589 papers was carefully analyzed to keep articles dealing with 3-D biomedical solid texture classification or retrieval. The references of each relevant publication were recursively traced back to extend the initial set of papers. A total

\footnotetext{
3 http://www.info.sciverse.com/scopus/, as of 9 October 2013.

4 http://portal.acm.org/, as of 9 October 2013.

5 http://www.ncbi.nlm.nih.gov/pubmed/, as of 9 October 2013.

${ }^{6}$ http://ieeexplore.ieee.org/Xplore/guesthome.jsp, as of 9 October 2013.

7 http://spiedigitallibrary.org/, as of 9 October 2013.

8 http://www.elsevier.com/, as of 9 October 2013.

9 http://www.springer.com/, as of 9 October 2013.
}

of 100 papers dealing with 3-D solid texture analysis in biomedical imaging was found (see Fig. 5). The number of papers has been increasing over the years and this is expected to continue because radiology services of modern hospitals are largely based on multidimensional images (Andriole et al., 2011). Since solid texture is difficult to visualize and to univocally describe in terms of human vocabulary, quantitative measures of biomedical textures are expected to provide powerful diagnosis tools in several imaging modalities.

\section{Imaging modalities}

The various volumetric data acquisition techniques used in the literature for solid texture analysis are briefly described in this section to better understand the characteristics of the data to be analyzed. The specifications of imaging modalities are compared in Table 1 in terms of signal measured, 3-D acquisition sequence, shape of the spatial transfer function, typical voxel size and image type. Most imaging modalities yield anisotropic 3-D lattices, where $\Delta z$ is larger than $\Delta x$ and $\Delta y$, and $\Delta x=\Delta y$. Re-slicing all images of a dataset using $\Delta z=\Delta x=\Delta y=\Delta_{\min _{D B}}{ }^{10}$ with 3-D image interpolation to obtain isotropic image resolution is required to ensure the conservation of scales and directions in all three dimensions. 2-D slice based texture analysis must be considered when $\Delta z \gg(\Delta x, \Delta y)$. Slice thickness has a strong influence on the texture appearance and must be consistent in the considered data collection (Savio et al., 2010; Smith et al., 2010; Packard et al., 2012).

\subsection{Magnetic resonance imaging (MRI)}

MRI measures nuclear magnetic resonance in a tomographic scanning sequence to create 3-D images. It provides therefore good contrast between soft tissue and is well-suited for imaging the brain, muscles, tumors and other tissue with high proton density. The tomographic acquisition sequence yields high and near-isotropic image resolutions. The pulse sequence (e.g., T1/T2-weighted) used can be tuned to achieve optimal tissue contrasts. Consequently, 3-D texture analysis in MRI has been most widely studied with 37 papers $(38.5 \%)$.

\subsection{X-ray computed tomography}

X-ray CT uses an X-ray beam in a tomographic helical scanning sequence to create a 3-D image of various body structures based on their X-ray absorption. It allows precise imaging of several anatomical structures and is widely used in clinical routine for diagnosing structural changes in the head, lungs, heart, abdomen, pelvic regions and extremities. It provides an optimal contrast to

\footnotetext{
${ }^{10} \Delta_{\min _{D B}}$ is the smallest voxel spacing over the database considered.
} 


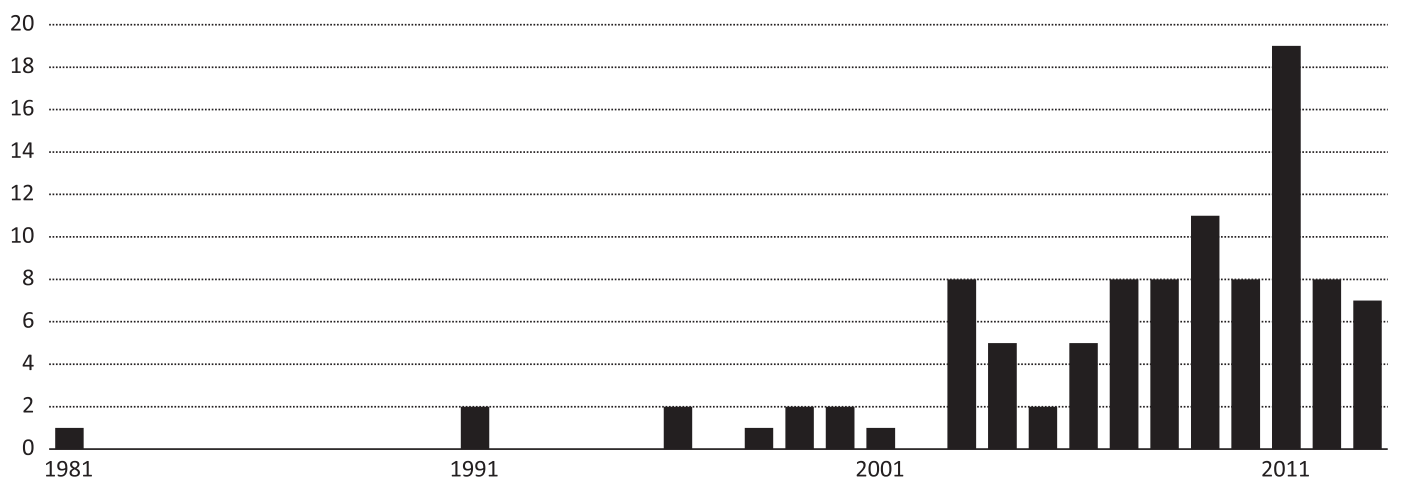

Fig. 5. Distributions of the 100 publications over the years.

Table 1

Specifications of imaging modalities.

\begin{tabular}{|c|c|c|c|c|c|c|}
\hline Modality & Signal measured & 3-D acquisition sequence & Spatial transfer function & Typical voxel size & Image type & \\
\hline MRI & $\begin{array}{l}\text { Nuclear magnetic } \\
\text { Reasonance }\end{array}$ & Tomographic & Ellipsoid & $\begin{array}{l}\Delta_{x}, \Delta_{y}: \\
\Delta_{z}:\end{array}$ & $\begin{array}{l}0.5-1 \mathrm{~mm} \\
0.5-5 \mathrm{~mm}\end{array}$ & Anatomical \\
\hline CT & X-ray absorption & Tomographic & Ellipsoid & $\begin{array}{l}\Delta_{x}, \Delta_{y}: \\
\Delta_{z}:\end{array}$ & $\begin{array}{l}0.5-1 \mathrm{~mm} \\
0.5-5 \mathrm{~mm}\end{array}$ & Anatomical \\
\hline Confocal & Optical & Exhaustive & Cylindrical & $\Delta_{x}, \Delta_{y}:$ & $\approx 0.4 \mu \mathrm{m}$ & Anatomical or \\
\hline Microscopy & Fluorescence & 3-D scanning & & $\Delta_{z}:$ & $\approx 0.8 \mu \mathrm{m}$ & Functional \\
\hline 3-D US & Acoustic reflection & Freehand & Undefined & $\Delta_{x}, \Delta_{y}, \Delta_{z}:$ & $0.5-1 \mu \mathrm{mm}$ & Anatomical \\
\hline$\mu \mathrm{CT}$ & X-ray absorption & Tomographic & Ellipsoid & $\Delta_{x}, \Delta_{y}, \Delta_{z}:$ & $\approx 1 \mu \mathrm{m}$ & Anatomical \\
\hline
\end{tabular}

image the lung and liver parenchyma, where texture information is most relevant to characterize tissue abnormalities.

\subsection{Confocal microscopy}

Unlike traditional wide-field fluorescence microscopes, confocal microscopy uses single point illumination and removes the entire out-of-focus signal to image a given point $(x, y, z) \in \mathbb{R}^{3}$. The precision of the illumination depends on the pointspread function imposed by the light source. Using a scanning sequence (i.e., a rectangular pattern of parallel scanning lines), a 3-D image can be obtained with a slice thickness that can be as thin as half a micron (Pawley, 2006). The characterization of subcellular structures, muscular fibers and capillary networks in confocal microscopy strongly relies on 3-D texture information.

\subsection{3-D ultrasound (US)}

Ultrasonography (US) measures the echo of emitted sound waves between 2 and $18 \mathrm{MHz}$. The delay between the sent wave and its echo determines the depth of the structures. An image can be created by assigning the amplitude of the echo to the pixel values. A combination of several waves emitted in complementary directions and computerized post-processing allows creating a three-dimensional US image. Scanning sequences in US are determined by the movements of the probe that the operator applies and are therefore not providing a complete overview of the organs and structures. As a consequence, shape-based features for image segmentation are hardly applicable in US. Relying on tissue appearance only, texture-based approaches are more robust for the segmentation of occluded or partial objects. High speckle noise levels in 3-D US require to use texture analysis approaches that are robust to noise.

\subsection{Micro-CT $(\mu C T)$}

Micro-CT, also called X-ray microtomography or $\mu \mathrm{CT}$, allows three-dimensional imaging of small structures with isotropic voxel resolutions of around $1 \mu \mathrm{m}$. This technique is well-suited to study the microstructures containing high calcium concentrations (e.g., bones or artery calcification).

\subsection{Positron emission tomography (PET)}

Positron emission tomography is a nuclear medical imaging technique that detects pairs of $\gamma$-rays emitted indirectly by a positron-emitting radionuclide (i.e., radiotracer), which is introduced into the body on a biologically active molecule. When the radio tracer used is fludeoxyglucose (FDG), the metabolism (i.e., glucose uptake) can be monitored in a given tissue to highlight active regions of the brain or cancer tumors. The spatial resolution of PET is low $\left(\Delta_{x}, \Delta_{y}, \Delta_{z}: 2-5 \mathrm{~mm}\right)$, which does not yield strong texture information in small regions.

\subsection{Optical coherence tomography (OCT)}

Optical coherence tomography leverages the principle of low coherence interferometry to generate images with a resolution smaller that $10 \mu \mathrm{m}$. Three-dimensional images are obtained with cross-sectional back-scattering profiles acquired in a tomographic sequence. The use of relatively long wavelengths allows penetrating deeper into the sample when compared to confocal microscopy. The high presence of noise is a major challenge when processing OCT images.

\section{Organs}

The distributions of the publications based on imaging modalities and organs are shown in Fig. 6, which reflects the complemen- 
tarity of texture and shape information. Organs where disorders are producing changes in tissue texture are well represented (e.g., brain, lung). Organs that are usually studied in terms of shape are observed at the microscopic scale, where texture information is most relevant (e.g., bones in $\mu \mathrm{CT}$, cardiac muscle fibers in confocal microscopy). The main image analysis tasks are: organ segmentation, lesion detection, classification and quantification. Texturebased phenotype identification is also proposed. Table 2 summarizes the geometries of the 3-D biomedical texture found in the literature.

\subsection{Brain}

Brain MRI is widely used in clinical routine and allows diagnosing and quantifying a wide range of neurological diseases and anomalies (Bradley et al., 2001). Brain tissue is generally categorized into gray and white matters as well as the cerebro-spinal fluid. The gray matter is composed of neuronal cell bodies and the white matter of their myelinated axons and glial cells. However, these microstructures $(\approx 50 \mu \mathrm{m})$ are not visible in MRI. The macrotexture information in the normal brain is rather homogeneous in the respective tissue types. Directional patterns are created by the gyrifications of the gray matter. Anomalies create local changes in the texture properties, which was exploited by several researchers to detect, segment and quantify brain lesions.

A discrimination between cerebro-spinal fluid, gray matter and white matter is carried out on the basis of 3-D texture properties in a wavelet representation in Barra and Boire (2000). The gyrifications of the gray matter create oriented spatial frequencies that are well captured by the 3-D multi-scale wavelet coefficients. The resulting proportions of segmented volumes are used to differentiate between Alzheimer's dementia, epilepsy, or hydrocephalus. 3-D texture analysis is used in El-Baz et al. (2008) to segment the white matter and further analyze the shape of its gyrifications as indicators of dyslexia. In Goldbach et al. (1991) and Yousefi et al. (2011), Markov random fields (MRF) are used to create probability density function maps of gray and white matter as well as cerebrospinal fluid. MRFs showed to be robust to the varying levels of speckle noise present in MRI.
The arbor vitae in the cerebellum also has a typical tree-like fractal texture pattern. Foncubierta-Rodríguez et al. (2012) used multi-scale 3-D Difference of Gaussian (DoG) wavelets to model this structure and segment the adult cerebellum.

Texture information was used by Shen and Bai (2008) as a measure of the registration quality of MRI images of the brain using the maximum response of 3-D Gabor wavelets.

Neurodegenerative diseases are associated with several structural alterations of the brain such as diffuse white matter hypointensities, periventricular hypointensities and enlarged periventricular spaces. Kovalev et al. (1999), Kovalev and Petrou (2000), and Kovalev et al. (2001) analyzed the intensity, gradient and anisotropy properties of 3-D texture in brain MRI for automatic detection of these diffuse anomalies. Li et al. (2011) extracted gray-level co-occurrence matrices (GLCM) and run-length matrices (RLE) texture features from the hippocampus and corpus callosum regions to discriminate Alzheimer's disease and mild cognitive impairment from normal controls. A similar study was performed by Li et al. (2010). In functional imaging, the heterogeneity of the FDG-PET signal in large areas of the brain is used for regionbased retrieval of similar patients affected by Alzheimer's disease, diffuse Lewy body disease and fronto-temporal dementia in Cai et al. (2010), Liu et al. (2011a), and Liu et al. (2011b).

Texture information was used to quantify structural brain asymmetry and anisotropic properties of the white matter by Kovalev et al. (2003a), Kovalev et al. (2003b), Kovalev and Kruggel (2007), and Kovalev and Petrou (2009). The authors could establish a correlation of the structural brain asymmetry with age and gender in Kovalev et al. (2003a), Kovalev and Kruggel (2007), and Kovalev and Petrou (2009), and discriminate between controls and schizophrenic patients in Kovalev et al. (2003b) and Kovalev and Petrou (2009).

Epileptogenic lesions create a local transient in brain texture, allowing for their detection in MRI (Antel et al., 2003). Contralateral comparisons of steerable 3-D wavelet coefficients were used by Toro et al. (2013) to provide local abnormality scores. A separable 3-D wavelet transform of the hippocampus is also used for automated detection of patients subject to temporal lobe epilepsy in Jafari-Khouzani et al. (2004). Subtle anomalies of the structures

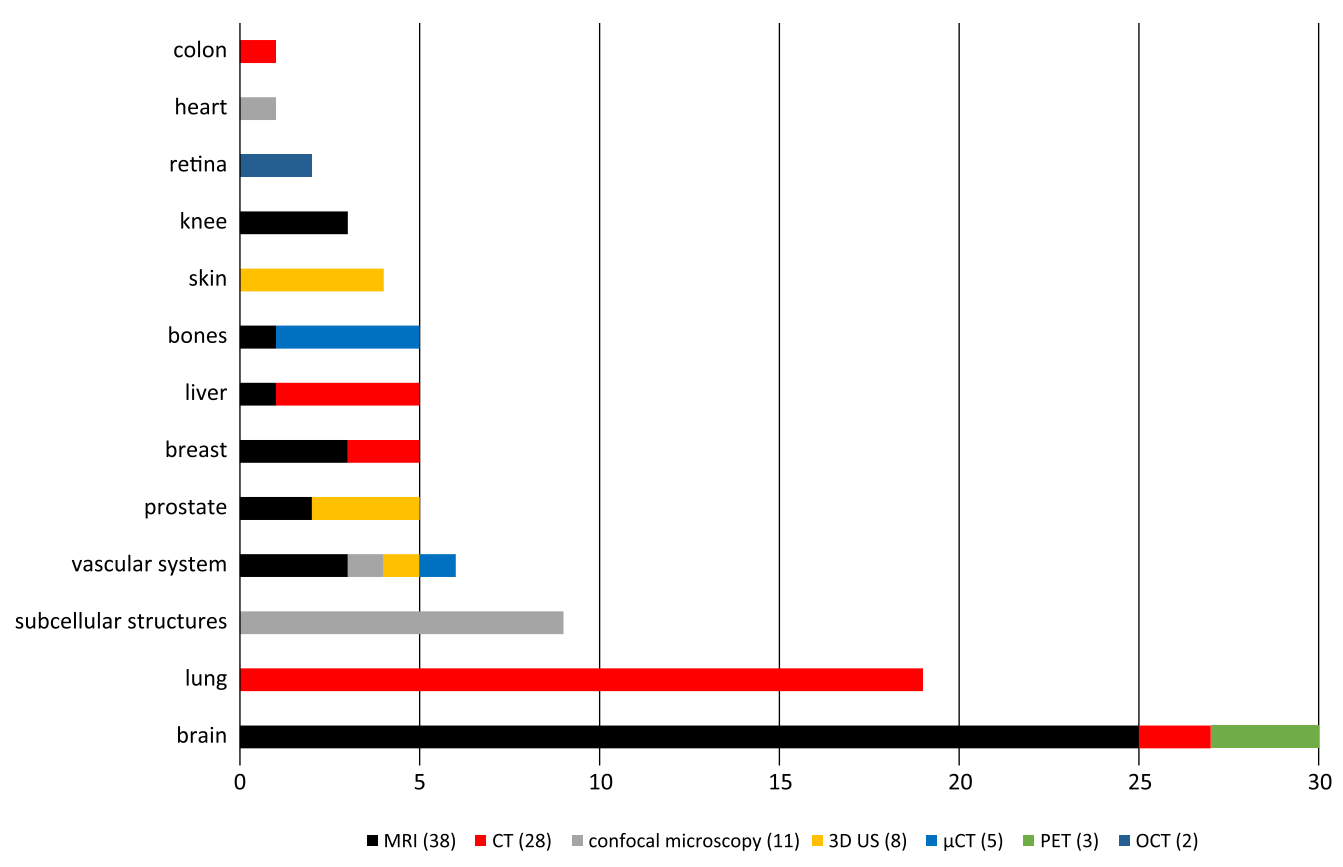

Fig. 6. Distributions of the publications based imaging modalities and organs. 
Table 2

Geometries of 3-D biomedical textures.

\begin{tabular}{ll}
\hline $\begin{array}{l}\text { 3-D texture } \\
\text { geometry }\end{array}$ & Examples \\
\hline Radial, isotropic & $\begin{array}{l}\text { Nodules, micronodules, hypo and 'hyper-intensities, } \\
\text { consolidation, PET signal, breast fat, cell nuclei }\end{array}$ \\
$\begin{array}{l}\text { Filaments, linear } \\
\text { structures }\end{array}$ & $\begin{array}{l}\text { Vessels, bronchus, lactiferous ducts, cancellous bone, } \\
\text { fibrosis } \\
\text { Burfaces }\end{array}$ \\
Arborescent & $\begin{array}{l}\text { heterogeneous tumors, skin layers, retinal layers } \\
\text { Vascular trees, bronchus, arbor vitae (cerebellum) }\end{array}$ \\
\hline
\end{tabular}

of deep gray matter in patients with progressive myoclonic epilepsy that could not be detected by direct visual inspection of the images in MRI were revealed by 3-D GLCMs, RLEs and gradients in Suoranta et al. (2013).

The internal texture of brain lesions was reported to correlate with the presence and type of cancerous cells. In Georgiadis et al. (2009) and Allin Christe et al. (2012), a discrimination between volumes of interest (VOIs) containing either metastases, gliomas and meningiomas using 3-D texture classification is carried out to assist brain cancer diagnosis. Texture features from 3-D GLCMs show improved classification performance of VOIs containing solid tumors, necrosis, edema, peritumoral regions, as well as homo-lateral and contra-lateral white matter when compared to traditional 2-D GLCMs in Mahmoud-Ghoneim et al. (2003). 3-D texture analysis of diffusion tensor (DT) MRI is carried out by Nunzio et al. (2011) to differentiate between healthy brain tissue and glioma tumors. Global multiple 3-D texture features are used in Gao et al. (2010) and Qian et al. (2011) to retrieve similar brains that are either normal or containing tumors.

Properties of brain texture in CT has been used to segment brain anomalies (Kovalev and Petrou, 1996) and to quantify traumatic brain injury (Ahmed and Farag, 1996).

\subsection{Lung}

The various appearances of healthy and altered lung parenchyma in high-resolution CT (HRCT) and multi-detector CT (MDCT) have typical texture signatures, which makes their automated recognition based on texture analysis reliable. This domain has been an important research topic during the past 15 years (Hoffman et al., 2003; Xu et al., 2006b; Depeursinge et al., 2012). Most of the studies focused on 2-D slice-based texture classification (Depeursinge et al., 2007; Depeursinge et al., 2011a), which was imposed by the inter-slice distance of $10 \mathrm{~mm}$ of the HRCT protocol (to keep radiation dose low). The introduction of the MDCT protocol in clinical routine allowed for 3-D solid texture analysis, thanks to its near-isotropic sub-millimetric voxel resolution.

The texture appearance in the lung parenchyma is strongly affected by the presence of bronchovascular structures. When compared to 2-D approaches, 3-D texture analysis can differentiate between isolated nodules and the intersection of a vascular structure and the projection plane. Comparisons between 2-D and 3-D approaches showed improved classification performance in Xu et al. (2005) and Xu et al. (2006b). The texture properties vary strongly with the anatomical location which was taken into account in only few articles (Depeursinge et al., 2011b and Depeursinge et al., 2013). The sections of the bronchus and vessel decrease with the distance to the mediastinum. The gravity has also an impact on the texture properties between the lung bases and apex.

Texture-based identification and characterization of interstitial pneumonia patterns and diffuse lung diseases in MDCT has been investigated by several research groups during the past 5 years
(Xu et al., 2005; Xu et al., 2006b; Xu et al., 2006a; Fetita et al., 2007a; Fetita et al., 2007b; Korfiatis et al., 2008; Boehm et al., 2008; Chang-Chien et al., 2009; Mariolis et al., 2010a; Korfiatis et al., 2010; Mariolis et al., 2010b; van Rikxoort et al., 2011). Interstitial and diffuse lung diseases create large regions of altered tissue (e.g., fibrosis, ground glass, emphysema, micronodules, consolidation) with well-defined texture properties, which are best described in terms of texture properties (Webb et al., 2001). 3-D texture analysis is also used to refine the vessel tree segmentation in presence of interstitial lung disease (Korfiatis et al., 2011) and other diffuse lung diseases (Korfiatis et al., 2009) in MDCT.

Pulmonary embolism (PE) and lung perfusion defects were related to the presence of oligemia, ground glass, mosaic perfusion and other texture-related patterns in CT (Karabulut and Kiroglu, 2008; Hansell et al., 2008). 3-D texture analysis in injected CT is carried out in Ganeshan et al. (2008) to quantify the extent of hypoperfused regions in PE. A similar approach is proposed by Depeursinge et al. (2013) to detect lung regions affected by PE in 4D dualenergy CT images. For each energy level in 40-140 keV, 3-D rotation-covariant wavelet features allow local alignment of the texture features on small vessels affected by ischemia. Based on similar data, Foncubierta-Rodríguez et al. (2013b) and Foncubierta-Rodríguez et al. (2013c) used bags of visual words from 3-D Laplacian of Gaussian (LoG) wavelets to characterize local pulmonary perfusion.

The presence of lung tumors creates local changes in the lung parenchyma and can be detected using texture. This is more challenging when compared to brain tumors, because the lung parenchyma is more heterogeneous. A combination of 2-D and 3-D texture features is investigated by Basu et al. (2011) for classifying between adenocarcinoma and squamous-cell carcinoma.

General lung CT retrieval based on texture was propose by Burner et al. (2012) using bags of visual words from 3-D local binary patterns.

\subsection{Subcellular structures}

Clusters of cell nuclei (e.g., pseudopalisading, lymphocytes), necrotic regions and the presence of proteins are all defining specific texture patterns in confocal microscopy. 3-D texture analysis can be used to automate the laborious segmentation of subregions, classify between benign and malignant cells, cancer cell grading, and even the discovery of image-based disease phenotypes.

The classification of cell nuclei in 3-D confocal imaging based on 3-D texture was investigated several times (Huisman et al., 2005; Fehr and Burkhardt, 2006; Huisman et al., 2007; Fehr, 2007; Fehr and Burkhardt, 2008; Kim and Choi, 2009; Kim et al., 2010). Huisman et al., 2005; Huisman et al., 2007 used a combination of GLCMs and fractal features to discriminate between benign and malignant prostate cell nuclei. 3-D texture features based on the phase of the Fourier transform on a sphere surrounding the voxel to be classified (i.e., phase of the spherical harmonics) with 3-D local binary patterns (LBP) are compared for the discrimination between 5 types of cell nuclei (erythrocyte, endothelia cells, pericyte, fibroblast and macrophage) in Fehr and Burkhardt (2006), Fehr (2007), and Fehr and Burkhardt (2008). Kim and Choi (2009) combine GLCMs with RLE matrices to grade renal cell carcinoma nuclei in confocal microscopy. A combination of these texture features with morphological features could improve grading accuracy in Kim et al. (2010).

Automated cell cycle phase classification in 3-D time-series image datasets of live Drosophila embryos is carried out by Du et al. (2011) using 3-D GLCMs combined with intensity and shape features. 3-D GLCMs are combined with morphological features in Chen and Murphy (2004) for the detection of various subcellular protein locations in cultured mammalian cells. 


\subsection{Vascular system}

Vascular trees in biomedical tissue have well-defined texture properties. They can be used to quantify the vascular density of tumors or the risk of stroke. Since vessels have typical filament and branching structures, texture approaches discarding the directional information are little used for their analysis. The section of these filament structures is varying, which motivates the use of directional fractals and multi-scale approaches.

In François et al. (2003), the responses of 3-D Gabor wavelets are used to measure local similarity between 3-D US images for intra- or inter-patient registration in the carotid region.

3-D GLCMs are used to analyze the architecture of vascular trees of rat brains in confocal microscopy in Kocinski et al. (2012) to characterize tumors. The density of small vessels (i.e., "vesselness", Sato et al., 1998) is used by Forkert et al. (2011) to assess the risk of stroke in MR angiography (MRA).

In large vessels or at a microscale, calcified regions and plaques have a well-defined texture appearance. In Alexander et al. (2007), 3-D texture features that are invariant to rigid transformations of the image (i.e., translation, rotation and scaling) are introduced for automated detection of calcified regions in the arteries in $\mu \mathrm{CT}$. Van Engelen et al. (2011) used 3-D Gaussian and Laplacian filters to classify carotid artery plaque in MRI to distinguish stable from vulnerable plaques.

\subsection{Prostate}

The prostate lobe parenchyma and its alterations can be characterized in 3-D US and MRI using texture. Akbari et al. (2011) and Yang et al. (2011) used 2-D wavelets in 3 orthogonal planes for the segmentation of the prostate in three-dimensional transrectal US images. Zhan and Shen (2006) used a Gabor filterbank for segmenting the prostate in 3-D US for cancer diagnosis, image-guided surgical planning and therapy. Lopes et al. (2011a) proposed automated prostate cancer detection in MR images using fractal analysis. Madabhushi et al. (2003) used 3-D Gabor filters to segment prostatic adenocarcinoma (MRI).

\subsection{Breast}

The breast parenchyma in MRI and X-ray digital breast tomosynthesis (CT approximation) has texture properties that are mainly modulated by fat heterogeneity, lobules and lactiferous ducts (i.e., directional filaments). The presence of ducts is higher in regions close to the nipple. The fat is mainly distributed in the periphery of the breast and the lobules in the center. Therefore, texture properties cannot be considered as homogeneous over the whole breast, which was not investigated in the literature.

Instead of considering the texture of the whole breast, breast mass classification and cancer detection using 3-D texture was studied. In Chen et al. (2007), 3-D GLCMs outperform their 2-D counterpart for the classification of benign versus malignant breast lesions in contrast-enhanced MRI, which is consistent with the findings of Mahmoud-Ghoneim et al. (2003). Breast tissue segmentation and density estimation from MRI using 3-D hidden Markov models is proposed by Shafer et al. (2011). Kontos et al. used solid texture analysis in three-dimensional X-ray digital breast tomosynthesis for both cancer detection in Kontos et al. (2009b) and cancer risk estimation in Kontos et al. (2009a).

\subsection{Liver}

Healthy liver parenchyma has a homogeneous 3-D texture appearance in CT and MRI. This creates the opportunity to both segment the liver from other organs and detect lesions based on deviations from the normal texture appearance. The latter was investigated by Upadhyay et al. (2012) using Gaussian Markov random fields (GMRF) that are invariant under 3-D-rotations and translations. Similarly to Depeursinge et al. (2013), aligning the texture instances along the directional structures (i.e., vessels) allows reducing the intra-class variation of the healthy tissue and therefore allow better detection of the lesions. Liver segmentation in CT and MRI was proposed by Kovalev and Petrou (1996), Philips et al. (2008), Danciu et al. (2012), and Lee and Strzelecki (2011), respectively.

\subsection{Bone}

Whereas little macrotexture information is found in bones in radiology images, 3-D microtextures are visible in cancellous bone cores at smaller scales in $\mu \mathrm{CT}$ and high-resolution MRI. Changes in the three-dimensional structure of the cancellous bone were often related to the diagnosis of osteoporosis (Odgaard, 1997; Mishra et al., 2011). In Showalter et al. (2006), a correlation of 3-D texture measures with manually established architectural indices of cancellous bone cores in $\mu \mathrm{CT}$ could be observed. Moreno et al. (2011) use local and global structure tensors to categorize bone microstructures as plate-, rod- and junction-like trabeculae. Ollivier et al. (2013) showed that the 3-D fractal dimension is an indicator of femoral neck fracture in $\mu \mathrm{CT}$ when combined with bone mineral density. In Alberich-Bayarri et al. (2010), a correlation of 2-D and 3-D fractal dimensions of the cancellous bone with osteoporosis and spinal bone mineral density was observed in high-resolution MRI, ${ }^{11}$ demonstrating that $3-\mathrm{D}$ texture analysis can assist clinicians in diagnosing osteoporotic patients.

\subsection{Skin}

Skin layers define homogeneous regions in 3-D US. Paulhac (2009), Paulhac et al. (2009b), Paulhac et al. (2009a), and Paulhac et al. (2009c) used a mixed 3-D texture feature set composed of 3-D separable wavelets, geometric measures based on binarization of the textures and statistical Tamura features (Tamura et al., 1978) to segment skin layers and tumors (i.e., histiocytofibroma).

\subsection{Knee}

Reyes-Aldasoro and Bhalerao (2003), Bhalerao and Reyes-Aldasoro (2003), and Reyes-Aldasoro and Bhalerao (2007) used 3-D multi-scale Gaussian filters with an octree orientation pyramid to classify tissue in MRI of the knee such as muscle, bone and other tissue.

\subsection{Heart}

Lang et al. (1991) use a three-dimensional Law's model to identify heart muscle fiber structures in 3-D confocal microscopic images.

\subsection{Retina}

Three-dimensional texture analysis of the retina in OCT images was investigated by Quellec et al. (2010) and Kafieh et al. (2013). Quellec et al. (2010) compared GLCMs, RLEs and wavelets for the identification of fluid-filled regions of the macula. Graph-based diffusion maps were used by Kafieh et al. (2013) to segment the intra retinal layers in OCT scans from normal controls and glaucoma patients.

\footnotetext{
${ }^{11} \Delta x=\Delta y=\Delta z=180 \mu \mathrm{m}$.
} 


\subsection{Whole body}

General segmentation of various organs and materials (e.g., liver, spleen, kidney, heart, stomach, backbone, fluids) based on their 3-D texture appearance in CT using 3-D Haralick texture features (i.e., measures derived from GLCMs) is investigated in Kurani et al. (2004) and Tesar et al. (2008) and with run-length encoding (RLE) in Xu et al. (2004).

\section{Techniques}

Depending on the nature of the data observed, geometric or stochastic modeling techniques enable automated texture analysis with varying success levels. Approaches based on simple primitives with small supports are modeling microtextures and refer to statistical methods, whereas complex primitives with large supports are referred to as structural or geometrical approaches in the literature (Reed and du Buf, 1993; Materka and Strzelecki, 1998). Approaches based on geometric models are likely to effectively describe textures when a priori knowledge is available (e.g., prevailing directions and scales, or templates). When little is known about the texture properties of the observed data, stochastic techniques allow exploring the statistical properties of the textural patterns. In this section, the techniques found in 100 papers dealing with biomedical 3-D solid texture analysis are reviewed, regrouped and categorized based on the type of texture information modeled by their set of primitives (see Tables 3 and 4). The approaches based exclusively on gray-levels and color distributions are not reviewed in this survey because they are not specific to 3-D data. The popularity of the various approaches is shown in Fig. 7. The techniques are qualitatively compared in Table 3 in terms of their primitive properties.

\subsection{Gray-level co-occurrence matrices (GLCM)}

Statistics of the values and relative positions of the pixels were among the earliest attempts of computerized texture description in two-dimensional images. The pioneer work of Haralick et al. (1973) is still widely used. It studies the statistics of the co-occurrence of pixels of gray-level values between pixel pairs separated by a given distance and direction that are defined by $(\Delta i, \Delta j)$. 3-D Co-occurrences between all possible gray-level values are then organized in gray-level co-occurrence matrices (GLCM)

$\boldsymbol{Q}_{\Delta i, \Delta j, \Delta k}(p, q)=\sum_{i=1}^{M} \sum_{j=1}^{N} \sum_{k=1}^{O} \operatorname{Cooc}(p, q, i, j, k, \Delta i, \Delta j, \Delta k)$,

where

$\operatorname{Cooc}(p, q, i, j, k, \Delta i, \Delta j, \Delta k)=$

$\{1, \quad$ if $F(i, j, k)=p$ and $F(i+\Delta i, j+\Delta j, k+\Delta k)=q$,

0 , otherwise.

from which several statistical measures (see Haralick et al. (1973) for exact definitions) can be derived and used as texture features (Haralick et al., 1973; Haralick, 1979).GLCMs are easily extended to $3-\mathrm{D}$ where the voxel pairs are defined by $(\Delta i, \Delta j, \Delta k)$.For each displacement parametrized by the triplet $(\Delta i, \Delta j, \Delta k)$, a co-occurrence matrix $\boldsymbol{Q}$ is built as explained in Eq.(1).The primitive associated to a triplet $(\Delta i, \Delta j, \Delta k)$ is depicted in Fig.8.Thanks to their conceptual simplicity, 3-D GLCMs ${ }^{12}$ were widely used for solid texture classification and retrieval in biomedical imaging (Kovalev and Petrou, 1996; Kovalev et al., 1999, 2001, 2003a,b; Madabhushi et al., 2003; Mahmoud-Ghoneim et al., 2003; Antel et al., 2003; Kurani et al.,

\footnotetext{
$\overline{12}$ also called Markovian analysis in Huisman et al. (2005), although not equivalent to Markov models.
}

2004; Chen and Murphy, 2004; Huisman et al., 2005; Xu et al., 2005, 2006b; Showalter et al., 2006; Huisman et al., 2007; Kovalev and Kruggel, 2007; Chen et al., 2007; Tesar et al., 2008; Korfiatis et al., 2008; Philips et al., 2008; Kim and Choi, 2009; Kovalev and Petrou, 2009; Korfiatis et al., 2009; Kontos et al., 2009b,a; Georgiadis et al., 2009; Korfiatis et al., 2010; Gao et al., 2010; Mariolis et al., 2010b; Kim et al., 2010; Cai et al., 2010; Li et al., 2010; Quellec et al., 2010; Qian et al., 2011; Korfiatis et al., 2011; Nunzio et al., 2011; Du et al., 2011; Basu et al., 2011; Li et al., 2011; Kocinski et al., 2012; Allin Christe et al., 2012; Lu et al., 2012; Suoranta et al., 2013). The choice of the parameters is crucial to obtain efficient texture descriptions. Finding parameters requires a priori knowledge on the observed data. The values of $(\Delta i, \Delta j, \Delta k)$ must be chosen to match important scales and directions in the volumetric data, which are difficult to guess in opaque volumetric datasets. The displacements must be chosen to approximate spherical neighborhoods for a fixed radius. A common practice is to exhaustively explore directions to span 13 uniformly distributed directions in the 3D Euclidean space. Small inter-voxel distance are often used (e.g., $\left.\sqrt{\Delta i^{2}+\Delta j^{2}+\Delta k^{2}} \in[1 ; 3]\right)$, as in Mahmoud-Ghoneim et al. (2003), Korfiatis et al. (2008, 2009), Kim and Choi (2009), Kontos et al. (2009b,a), Georgiadis et al. (2009), Korfiatis et al. (2010), Mariolis et al. (2010b), Kim et al. (2010), Cai et al. (2010), Korfiatis et al. (2011), and Allin Christe et al. (2012). The size of the matrices $\boldsymbol{Q}$ is determined by the number of gray levels considered, and a reduction to 32,16 or 8 bits is often required to limit their dimensions and sparsity.

Basic rotation-invariance can be obtained by summing all GLCMs from the 13 directions (Chen et al., 2007) or by averaging the statistical measures as carried out in Antel et al. (2003). To avoid discarding relevant directional information, Philips et al. (2008) are extracting prevailing texture directions by using principal component analysis on the GLCM statistical measures organized in a way that each column represents a direction. Higher level features are proposed by Ip and Lam (1994) where the relationships between GLCM statistics in 3-D regions defined by octree structures are studied using 3-D adjacency graphs.

\subsection{Run-length encoding (RLE)}

Another popular technique based on gray-level spatial dependencies that was extended to $3-D$ is the run-length encoding (RLE) analysis proposed by Galloway (1975). A gray-level run is a sequence of consecutive, collinear voxels having the same gray level value. For a given direction, a matrix $\boldsymbol{Q}(m, l)$ can be built by storing the number of times that the picture contains a run of length $l$, consisting of collinear voxels having gray level $m$ oriented along a direction $(\Theta, \Phi)$ (see Eq. (2)).

$\boldsymbol{Q}_{\Theta, \Phi}(m, l)=\left|\operatorname{Runs}_{\Theta, \Phi}(m, l)\right|$,

where $\operatorname{Runs}_{\Theta, \Phi}(m, l)$ is the set of runs of length $l$, gray level $m$ and oriented along $(\Theta, \Phi)$. An example of a RLE primitive is shown in Fig. 9. Several statistical measures can be extracted from this matrix to quantify textures (see Galloway, 1975 for a list of the measures and their mathematical definitions). RLEs require similar gray-level reductions and choice of directions as GLCMs. However, the lengths $l$ of the runs are modeling texture scales and RLEs are therefore able to study textures containing several dominant scales. RLEs were extended to 3-D in Xu et al. (2004, 2005, 2006b,a), Kim and Choi (2009), Georgiadis et al. (2009), Mariolis et al. (2010a,b), Li et al. (2010), Kim et al. (2010), Quellec et al. (2010), Nunzio et al. (2011), Basu et al. (2011), and Li et al. (2011) and have the same risks of missing important information as do GLCMs when carrying out the required choices of the gray-level reductions and directions. 


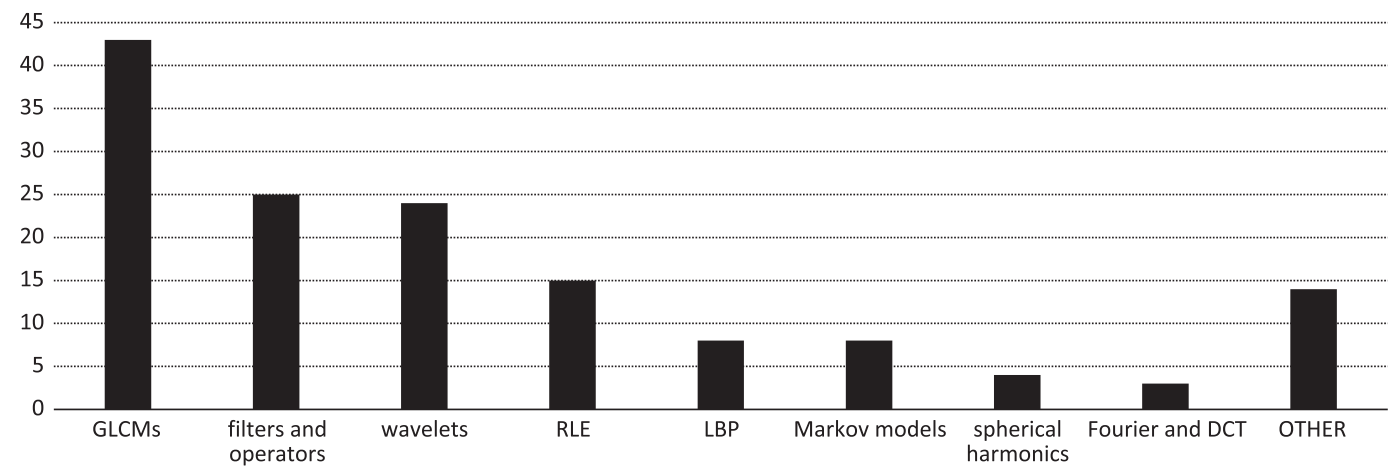

Fig. 7. Distributions of the publications based 3-D texture analysis approaches. Some papers are using several techniques and are represented in several bars.

\subsection{Local Binary Patterns (LBP)}

The statistics of the spatial organization of voxels on the surface of spherical neighborhoods is studied by extending Ojala's local binary patterns (Ojala et al., 2002) to 3-D in Fehr (2007), Fehr and Burkhardt (2008), Paulhac et al. (2008), Gao et al. (2010), Qian et al. (2011), Liu et al. (2011b), Mishra et al. (2011), Burner et al. (2012), and Venkatraghavan and Ranjan (2012). LBPs describe the organization of binarized voxels over spherical neighborhoods with sequences of bits $\mathrm{LBP}_{P, R}$. For a spherical neighborhood of radius $R$ containing $P$ equally spaced points over $(\Theta, \Phi)$, 3-D LBPs are defined as:

$\operatorname{LBP}_{P, R}=\sum_{p=0}^{P-1} s\left(m_{p}-m_{c}\right) 2^{p}$,

where

$s(x)= \begin{cases}1, & \text { if } x \geqslant 0 \\ 0, & \text { otherwise }\end{cases}$

$m_{c}$ and $m_{p}$ correspond to the gray-level values of the center and peripheral voxels respectively. $s(x)$ yields illumination-invariant binary spherical neighborhoods. A texture is characterized by the frequencies of the sequences of bits $\mathrm{LBP}_{P, R}$. An example of a LBP primitive is shown in Fig. 10. Rotation-covariant LBPs are obtained by exhaustively performing circular bit-wise right shifts on the sequences $\mathrm{LBP}_{P, R}$. The ordering of $P$ points is straightforward in 2-D on circular neighborhoods, but is undefined in 3-D. Fehr and Burkhardt (2008) proposed to define an arbitrary ordering over $(\Theta, \Phi)$ and to use it for all primitives. Burner et al. (2012) used cylindrical neighborhoods by concatenating 2-D LBPs along axis $K$. LBPs are grayscale invariant and characterize the spherical frequencies and structures in a neighborhood defined by $R$. They are therefore related to spherical harmonics and characterize the relationships between the local directions in textures. The scaling parameter $R$ and the parameter $P$ require optimization to successfully apply 3-D LBPs to a given solid texture analysis problem. Fehr (2007) and Fehr and Burkhardt (2008) developed fast computation of 3-D LBPs using spherical harmonics and the fast Fourier transform. $P$ relates to the spherical frequencies of spherical harmonics. The choice of the scales related to the radiuses $R$ of the concentric spheres is an important optimization problem of this method.

$$
\begin{aligned}
G(i, j, k) & =F * \Psi(i, j, k) \\
& =\sum_{m=-M_{\Psi}}^{M_{\Psi}} \sum_{n=-N_{\Psi}}^{N_{\Psi}} \sum_{0=-O_{\Psi}}^{O_{\Psi}} F \cdot \Psi(i-m, j-n, k-0) .
\end{aligned}
$$

\subsection{Convolutional approaches and spectral characterization}

A large group of approaches use discretized functions $\Psi(i, j, k)$ of support $\left(M_{\Psi} \times N_{\Psi} \times O_{\Psi}\right)$ as primitives. The 3-D signal can then be convolved with the set of primitives to render a new representation as explained in Eq. (5). In particular, the convolution yields response maps $G(i, j, k)$ that quantify the presence of the primitive in the 3-D texture. When the set of primitives is constituted by oscillatory functions at different scales, the texture analysis approach allows characterizing the spatial spectrum (i.e., distribution of scales) of the signal. The characterization of the spatial periodicities and regularities of the textures at various scales has been commonly used in 2-D texture analysis and was also extended to 3-D. Spectral descriptions of textures have the advantage of being multi-scale (i.e., they can continuously characterize changes in scales) and often gray-level invariant. In spectral analysis, any signal can be univoquely expressed in terms of its frequency spectrum. Whereas several approaches are suitable to approximate the frequency spectrum, all are relying on convolutions of the signal with a set of oscillatory functions (i.e., primitives) of different scales and phases. The Fourier and non-redundant wavelet transforms are providing access to the linear spatial spectrum in a given direction and allow for perfect reconstruction. Spherical harmonics allow an overcomplete characterization of the spherical spatial spectrum over a sphere of radius $R$. When a priori knowledge concerning the important scales that are contained in the observed texture is available, filters and filterbanks can provide adequate approximations of the specific spectral signatures of the considered texture patterns.

\subsubsection{Separability of the 3-D convolution}

Under certain conditions, $\Psi(i, j, k)$ can be separated into three 1-D functions $\Psi_{1}(i), \Psi_{2}(j)$ and $\Psi_{3}(k)$ of supports $\left(M_{\Psi} \times 1 \times 1\right)$, $\left(1 \times N_{\Psi} \times 1\right)$ and $\left(1 \times 1 \times O_{\Psi}\right)$ as

$\Psi(i, j, k)=\Psi_{1}(i) \otimes \Psi_{2}(j) \otimes \Psi_{3}(k)$.

By combining Eqs. (6) and (5), the convolution of $\Psi$ and $F$ becomes Eq. (7). The complexity of the convolution of a $M \times M \times M$ volume with a $N \times N \times N$ filter decreases from $\mathcal{O}\left(M^{3} N^{3}\right)$ to $\mathcal{O}\left(M^{3} \cdot 3 N\right)$. Unfortunately, the functions $\Psi_{1}, \Psi_{2}$ and $\Psi_{3}$ have uneven angular responses and are strongly biased towards directions $I$, J, and $K$ (Unser and Van De Ville, 2010; Chenouard and Unser, 2012). They do not allow for an isotropic characterization of the 3-D directions.

$G(i, j, k)=\Psi_{3}(k) *\left(\Psi_{2}(j) *\left(\Psi_{1}(i) * F\right)\right)$.

$\widehat{F}(u, v, w)=\sum_{m=0}^{M-1} \sum_{n=0}^{N-1} \sum_{o=0}^{O-1} F(m, n, o) e^{-j\left(\frac{2 \pi}{M}\right) u m} e^{-j\left(\frac{2 \pi}{N}\right) v n} e^{-j\left(\frac{2 \pi}{0}\right) w o}$.

where $j$ is the imaginary unit.

\subsubsection{Fourier analysis and discrete cosine transform (DCT)}

The Fourier analysis relies on the property that every signal can be approximated by a linear combination of sine and cosine functions in a given direction as defined in Eq. (8). This is equivalent to the convolution of $F(i, j, k)$ with sine and cosine functions. The func- 


\begin{tabular}{|c|c|c|c|c|c|}
\hline Technique & Example of primitive & Primitive neighborhood & Illumination invariance & Typical coverage of 3-D directions & Typical spatial spectrum coverage \\
\hline GLCMs & & Voxel pairs & No & Incomplete for $R>1$ & Incomplete \\
\hline RLE & & Linear & No & Incomplete for $R>1$ & Incomplete \\
\hline Separable Fourier transform and DCT & & Linear & Yes & Complete, non-isotropic & Complete, but non-local analysis \\
\hline Separable filters and wavelets & & Linear & Yes & Complete, non-isotropic & Filters: incomplete, wavelets: complete. \\
\hline LBP & 4 & Spherical & Yes & Complete, isotropic & Incomplete \\
\hline Spherical harmonics & & Spherical & Yes & Complete, isotropic & Incomplete \\
\hline Markov models & & Unconstrained & No & Incomplete & Incomplete \\
\hline Non-separable filters and wavelets & & Unconstrained & Yes & Complete, non-isotropic (isotropic for steerable wavelets) & $\begin{array}{l}\text { Filters: incomplete, } \\
\text { wavelets: complete }\end{array}$ \\
\hline
\end{tabular}




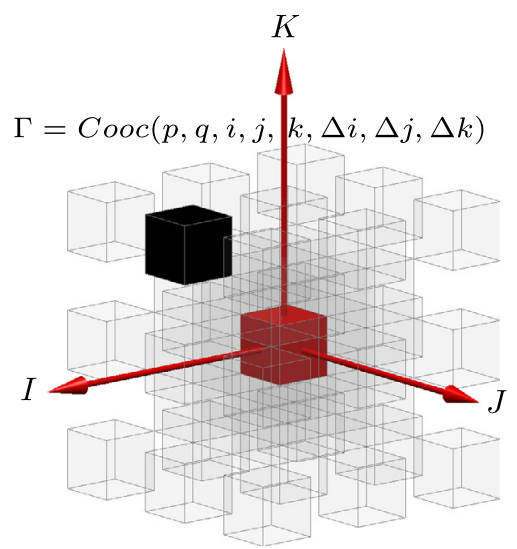

Fig. 8. GLCM primitive for $\Delta i=2, \Delta j=0, \Delta k=2$.

tions with highest factors are representative of the spectral signature of the signal. An example of a Fourier primitive is shown in Fig. 11. Since the Fourier transform is based on non-locally supported functions, it yields global measures of the frequency spectrum and is not adapted to characterize texture properties in a localized region of the image. To overcome this limitation, a common practice is to compute the Fourier transform in a local region of interest (ROI), which is also called "windowed" Fourier transform, where the boundary conditions are specified by a given window function. Such a technique is used by Kontos et al. (2009a,b) to estimate the fractal dimension in 3-D regions of interest (ROI). Directions are uniformly sampled to obtain 24 azimuthal and 12 elevation directions. Unfortunately, a separable Fourier transform is used, which biases the angular responses towards the main image axes. The discrete cosine transform (DCT) is similar to the Fourier transform but uses only the cosine function (i.e., real values). It is used by Danciu et al. (2012) in sliding windows to characterize local solid texture properties.

\subsubsection{Filters and operators}

Instead of using a windowed Fourier transform, local spectral properties can be obtained by convolving the signal with a given template $\Psi$ (i.e., primitive), which corresponds to filtering theory. The template or filter is a function of limited support $\left(M_{\Psi} \times\right.$ $\left.N_{\Psi} \times O_{\Psi}\right)$ that has given directional, scale and phase properties. Separability is also available when $\Psi$ satisfies the required conditions (see Eq. (6)), and have the same drawback of yielding anisotropic analysis. Separable and non-separable filter primitives are compared in Fig. 12.

The primitive $\Psi$ can be designed to detect specific features in 3D such as edges, ridges, corners and other structures. The 3-D edge detector proposed by Zucker and Hummel (1981) was used for solid texture analysis by Kovalev et al. (1999), Kovalev and Petrou (2000), Kovalev et al. (2001, 2003a,b), Kovalev and Kruggel (2007), and Kovalev and Petrou (2009) to estimate the 3-D gradient and study anisotropic properties of solid textures. This approach characterizes the important directions in the images but lacks multi-scale representation. Such multi-scale representation can be achieved by defining the 3-D edge operator of Zucker and Hummel (1981) at various scales. Gradients are also used to study prevailing directions of textures and can be computed from 3-D Gaussian filters (Madabhushi et al., 2003; Antel et al., 2003). In Madabhushi et al. (2003), normalized image gradients in $I, J$ and $K$ directions are obtained by applying separate derivations along the axes of the image.

Laws filters that are constructed from exhaustive combinations of separable step, edge, spot, ripple and wave detectors are extended to 3-D in Lang et al. (1991), Suzuki et al. (2006, 2009), Mariolis et al. (2010a), and Basu et al. (2011). They rely on the assumption that important features in textures are resulting from arbitrary combination of the above-mentioned objects.

2-D Gabor filters are modeling neurophysiological measurements of the two-dimensional anisotropic receptive field profiles describing single neurons in the mammalian visual cortex (Jones and Palmer, 1987). Gabor filters are therefore well-suited for texture representation and discrimination. Gabor filterbanks were ex-

Table 4

Detailed list of references per technique.

\begin{tabular}{|c|c|}
\hline Techniques & Papers \\
\hline $\begin{array}{l}\text { Connected components, blobs and } \\
\text { watersheds }\end{array}$ & Fetita et al. (2007a,b), Paulhac et al. (2009b,a), Paulhac (2009), Paulhac et al. (2009c), and Chang-Chien et al. (2009) \\
\hline Minkowski functionals & Boehm et al. (2008) and van Rikxoort et al. (2011) \\
\hline Fractals & Xu et al. (2005, 2006b,a), Alberich-Bayarri et al. (2010), and Ollivier et al. (2013) \\
\hline Markov random fields & $\begin{array}{l}\text { Goldbach et al. (1991), Ranguelova and Quinn (1999), El-Baz et al. (2008), Shafer et al. (2011), Yousefi et al. (2011), Lee and } \\
\text { Strzelecki (2011), Upadhyay et al. (2012), and Kafieh et al. (2013). }\end{array}$ \\
\hline GLCM & $\begin{array}{l}\text { Kovalev and Petrou (1996), Kovalev et al. (1999, 2001, 2003a,b), Madabhushi et al. (2003), Mahmoud-Ghoneim et al. (2003), Antel } \\
\text { et al. (2003), Chen and Murphy (2004), Kurani et al. (2004), Huisman et al. (2005), Xu et al. (2005, 2006b), Showalter et al. (2006), } \\
\text { Huisman et al. (2007), Kovalev and Kruggel (2007), Chen et al. (2007), Tesar et al. (2008), Philips et al. (2008), Korfiatis et al. (2008, } \\
\text { 2009, 2010, 2011), Kovalev and Petrou (2009), Kim and Choi (2009), Kontos et al. (2009b,a), Georgiadis et al. (2009), Cai et al. } \\
\text { (2010), Kim et al. (2010), Mariolis et al. (2010b), Gao et al. (2010), Li et al. (2010), Quellec et al. (2010), Nunzio et al. (2011), Du } \\
\text { et al. (2011), Basu et al. (2011), Li et al. (2011), Qian et al. (2011), Kocinski et al. (2012), Allin Christe et al. (2012), Lu et al. (2012), } \\
\text { and Suoranta et al. (2013) }\end{array}$ \\
\hline RLE & $\begin{array}{l}\text { Xu et al. (2004, 2005, 2006b,a), Kim and Choi (2009), Georgiadis et al. (2009), Kim et al. (2010), Mariolis et al. (2010a,b), Li et al. } \\
\text { (2010), Quellec et al. (2010), Nunzio et al. (2011), Basu et al. (2011), Li et al. (2011), and Suoranta et al. (2013) }\end{array}$ \\
\hline LBP & $\begin{array}{l}\text { Fehr (2007), Fehr and Burkhardt (2008), Gao et al. (2010), Qian et al. (2011), Liu et al. (2011b), Mishra et al. (2011), Burner et al. } \\
\text { (2012), and Venkatraghavan and Ranjan (2012) }\end{array}$ \\
\hline Fourier and DCT & Kontos et al. (2009b,a) and Danciu et al. (2012) \\
\hline Spherical harmonics & Fehr and Burkhardt (2006), Alexander et al. (2007), Fehr (2007), and Fehr and Burkhardt (2008) \\
\hline Filters and operators & $\begin{array}{l}\text { Zucker and Hummel (1981), Lang et al. (1991), Ahmed and Farag (1996), Sato et al. (1998), Kovalev et al. (1999, 2001, 2003a,b), } \\
\text { Kovalev and Petrou (2000, 2009), Reyes-Aldasoro and Bhalerao (2003), Bhalerao and Reyes-Aldasoro (2003), Madabhushi et al. } \\
\text { (2003), Antel et al. (2003), Kovalev and Kruggel (2007), Reyes-Aldasoro and Bhalerao (2007), Ganeshan et al. (2008), Mariolis et al. } \\
\text { (2010a), Gao et al. (2010), Qian et al. (2011), Forkert et al. (2011), Moreno et al. (2011), Van Engelen et al. (2011), Basu et al. } \\
\text { (2011), and van Rikxoort et al. (2011) }\end{array}$ \\
\hline Wavelets & $\begin{array}{l}\text { Barra and Boire (2000), François et al. (2003), Luche et al. (2004), Jafari-Khouzani et al. (2004), Zhan and Shen (2006), Shen and Bai } \\
\text { (2008), Paulhac et al. (2009b,a,c), Paulhac (2009), Gao et al. (2010), Quellec et al. (2010), Qian et al. (2011), Akbari et al. (2011), } \\
\text { Yang et al. (2011), Lopes et al. (2011a), Basu et al. (2011), Liu et al. (2011a), Venkatraghavan and Ranjan (2012), Foncubierta- } \\
\text { Rodríguez et al. (2012, 2013b,c), Depeursinge et al. (2013), and Toro et al. (2013) }\end{array}$ \\
\hline
\end{tabular}




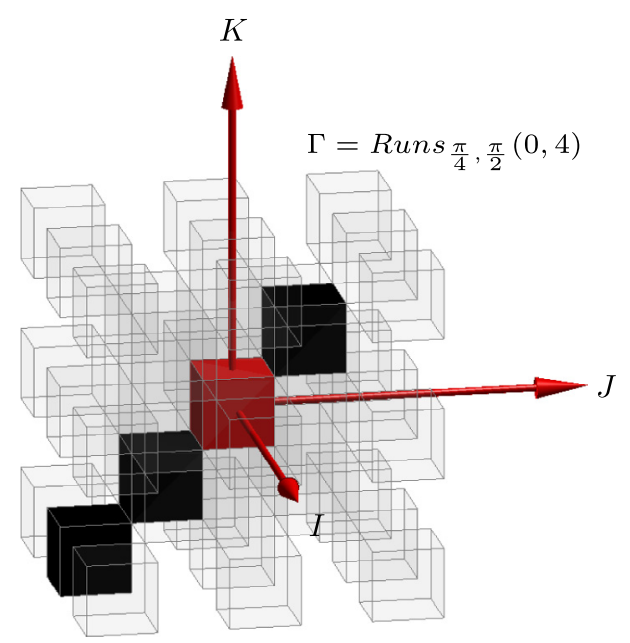

Fig. 9. RLE primitive of length $l=4$, gray level $m=0$ and oriented along $\left(\Theta=\frac{\pi}{4}, \Phi=\frac{\pi}{2}\right)$.

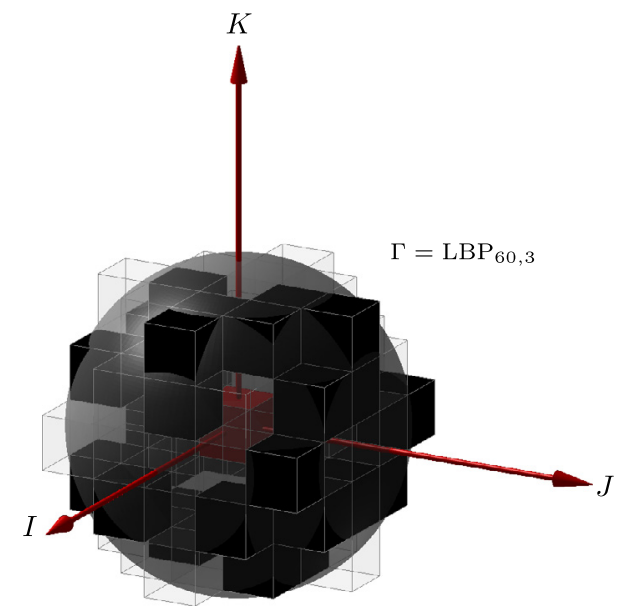

Fig. 10. LBP primitive of radius $R=3$ and all surface points considered $(P=60)$.

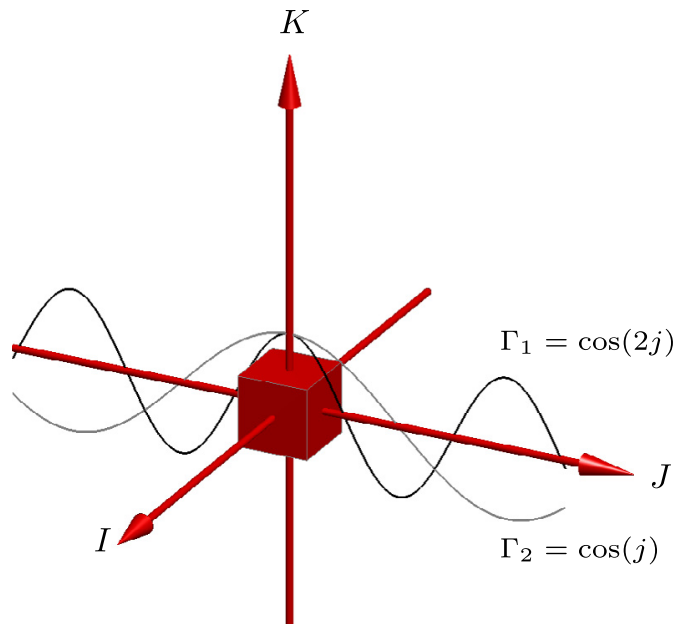

Fig. 11. Separable Fourier primitives along $J$.

tended to three dimensions in Bigun (1994), Madabhushi et al. (2003), Gao et al. (2010), and Qian et al. (2011) and require optimization of the scale and orientation parameters to the considered 3D textures.
A systematic approach for the tessellation of the 3-D frequency domain (i.e., the choice of scales and directions) is proposed by Reyes-Aldasoro and Bhalerao (2003), Bhalerao and Reyes-Aldasoro (2003), and Reyes-Aldasoro and Bhalerao (2007) by using a 3-D orientation pyramid, which is an extension of the bi-dimensional Wilson-Spann subband filtering (Wilson and Spann, 1988).

Marr and Hildreth (1980) suggested that the raw primal sketch in human vision (i.e., pre-attentive vision) is constituted by a collection of images filtered with 2-D Laplacian of Gaussian (LoG) at various scales, in which the zero-crossings are detected. LoG have the desirable property of providing well-defined band-pass filtering allowing to control the tessellation of the spectrum. Subsequently, LoG were also extended to 3-D for solid texture analysis in Ahmed and Farag (1996), Ganeshan et al. (2008), Van Engelen et al. (2011), van Rikxoort et al. (2011), Foncubierta-Rodríguez et al. (2012, 2013b,c).

Suzuki, 2007 developed a 3-D isotropic filter implementing the Hurst operator to estimate the fractal dimension at a given position and scale. They used a template bank in Suzuki et al. (2004b,a) to estimate the 3-D high order local autocorrelation by multiplying the 3-D textures with randomly positioned templates.

A "vessel filter" is proposed by Sato et al. (1998) as the response of multi-scale 3-D Hessian matrices. Hessian matrices implement second-order local derivatives of the 3-D image, and the associated eigenvalues describe the types of local underlying structures (e.g., line, plane). Similarly, eigenvalues of 3-D structure tensors are used by Moreno et al. (2011) to characterize local 3-D bone structures in $\mu \mathrm{CT}$ imaging.

\subsubsection{Wavelets}

Filterbanks can under certain conditions cover the whole spectrum of the image and are so-called wavelets (Mallat, 1999). Wavelet transforms (WT) are filterbanks constituted by oscillatory functions with various scale progressions ranging from high to low frequencies and a scaling function encompassing the lowest frequencies as defined in Eq. (9). WTs are providing systematic multi-scale descriptions of the signals when the scaling parameters $a$ are following a monotonous scale-progression (e.g., dyadic). Eq. (9) consists of convolving the signal with wavelet filters of varying scales in the continuous domain. The admissibility condition for $\psi$ as wavelet is to have a zero mean:

$w_{a}(x, y, z)=\int_{-\infty}^{\infty} \int_{-\infty}^{\infty} \int_{-\infty}^{\infty} f \frac{1}{\sqrt{a}} \psi\left(\frac{x-t}{a}, \frac{y-u}{a}, \frac{z-v}{a}\right)$.

$\int_{-\infty}^{\infty} \int_{-\infty}^{\infty} \int_{-\infty}^{\infty} \psi(x, y, z) d x d y d z=0$.

This also ensures that the wavelet coefficients $w_{a}(x, y, z)$ are invariant to uniform changes in illumination. The shape of wavelet primitives is only constrained by the admissibility condition (see Eq. (10)). Examples of wavelet primitives can be seen in Fig. 12.

Wavelets were used for 3-D texture analysis in Barra and Boire (2000), Jafari-Khouzani et al. (2004), Paulhac et al. (2009b,a,c), Paulhac (2009), Gao et al. (2010), Qian et al. (2011), Akbari et al. (2011), Yang et al. (2011), Lopes et al. (2011a), and Basu et al. (2011) in the separable form. Separability allows computationally effective WTs but has the drawback of characterizing textures along the image axes, only. To obtain a trade-off between computational complexity and orientation characterization, Zhan and Shen (2006) used 2-D Gabor filterbanks in two orthogonal planes. Because of their ability to describe 3-D textures in any direction, non-separable WTs were also used for solid texture analysis. Non-separable 3-D Gabor wavelets, which are constituted by a filterbank of Gabor templates with a given scale-progression (e.g., dyadic, quincunx) are used in François et al. (2003) and Shen and Bai (2008). Although being able to characterize any direction 

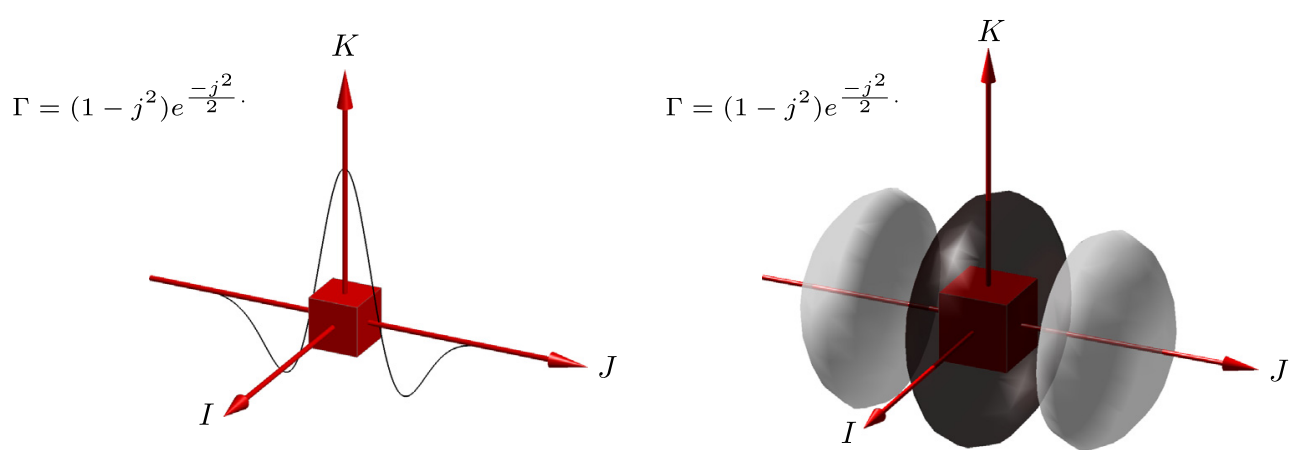

Fig. 12. Primitive from Laplacians of Gaussian along J. Left: separable versus right: non-separable convolution.

$(\Theta, \Phi)$, the directions of non-steerable directional WTs need to be explicitly formulated. This again requires arbitrary choices on the directions. Curvelets were introduced as a way of comprehensively partitioning the angular space (Candès and Donoho, 2000) and were extended to 3-D by Ying et al. (2005). Curvelets were used by Liu et al. (2011a) for the characterization of 3-D texture in FDG-PET images. An interesting alternative is to use steerable filterbanks, allowing a continuous characterization of the orientation space (Freeman and Adelson, 1991; Jacob and Unser, 2004). Steerable filterbanks allow analytically obtaining filter coefficients at any arbitrary orientation from a linear combination of the basis filters. This concept was used with wavelet filterbanks (e.g., the steerable pyramid, Simoncelli and Freeman, 1995) enabling multi-scale and isotropic multi-orientation analysis with optimal angular precision. The steerable pyramid was extended to 3-D in Luche et al. (2004) and Aguet et al. (2005) for the detection of directional structures but contributions using steerable wavelets for 3-D solid texture characterization are scarce (Depeursinge et al., 2013; Toro et al., 2013). Depeursinge et al. (2013) used 3-D steerable filters to align the filters along vessel structures in pulmonary CT, which showed to significantly improve the quantification of local perfusion.

\subsubsection{Spherical harmonics}

The spherical harmonics represent an interesting alternative to sine, cosine and other planar oscillatory functions to study the spatial frequencies in a 3-D Euclidean space. Spherical harmonics are the counterparts of Fourier basis functions on the sphere. They are adapted to study the local spherical spectrum in spherical neighborhoods and are therefore closely related to 3-D LBPs (Fehr, 2007; Fehr and Burkhardt, 2008). The spherical neighborhood $F(\Theta, \Phi)$ of of point $(i, j, k)$ can be represented by the sum of spherical harmonics $Y_{l}^{m}(\Theta, \Phi)$ as:

$F(\Theta, \Phi)=\sum_{l=0}^{\infty} \sum_{m=0}^{l} a_{l m} Y_{l}^{m}(\Theta, \Phi)$.

where $l$ denotes the band of expansion, $m$ the number of components for the $l$ th band and $a_{l m}$ the harmonic coefficient. The harmonic base functions $Y_{l}^{m}$ are calculated as:

$Y_{l}^{m}(\Theta, \Phi)=\sqrt{\frac{2 l+1}{4 \pi} \frac{(l-m) !}{(l+m) !}} L_{l}^{m}(\cos (\Theta)) e^{(j m \Phi)}$,

where $L_{l}^{m}$ is the Legendre polynomial and $j$ is the imaginary unit. An example of a spherical harmonic primitive is shown in Fig. 13.

Similarly to 3-D LBPs, the characterization of the directions is encompassed by the choice of the functions and has no straightforward interpretation. Fehr and Burkhardt (2006) introduce multiscale and rotation covariant 3-D texture features based on the relationships between the phases of spherical harmonics of the same band but from various concentric spherical neighborhoods. Rotation, scale and translation covariance are also achieved by Alexander et al. (2007) and Upadhyay et al. (2012), where the tensor product of multi-scale Gaussian radial functions with spherical harmonics yields steerable feature maps. The two latter approaches (i.e., Fehr and Burkhardt, 2006; Alexander et al., 2007; Upadhyay et al., 2012) are powerful tools to explore textures when no a priori knowledge is available on prevailing locations, scales and directions, which is often the case with solid 3-D textures.

\subsection{Markov probabilistic models}

Markov random fields (MRF) model the probability of a given voxel sequence (i.e., the primitive) using undirected graphs. MRFs are assumed to obey to symmetric autoregressive models defined by a given conditional probability density function. An example of a MRF primitive is shown in Fig. 14. 3-D hidden Markov models are used by Shafer et al. (2011) to learn the probability of voxel sequences associated with a given class in MRI. Upadhyay et al. (2012) use simple order-one Gaussian Markov random fields (GMRF) to model the orbits of 3-D texture rotations, yielding features invariant to rigid transformations (see Jain et al., 2012).

Relationships between gray-level values of voxels in volumetric spherical neighborhoods are encoded using 3-D GMRF by Ranguelova and Quinn (1999). The authors also provide an approach for the estimation of the GMRF parameters based on least squares. In Goldbach et al. (1991), GMRF parameters are obtained based on parametric Bayesian estimation. Reversible Markov chain analysis of topological graphs is used in Kafieh et al. (2013) to form diffusion maps. The corresponding primitive geometry is

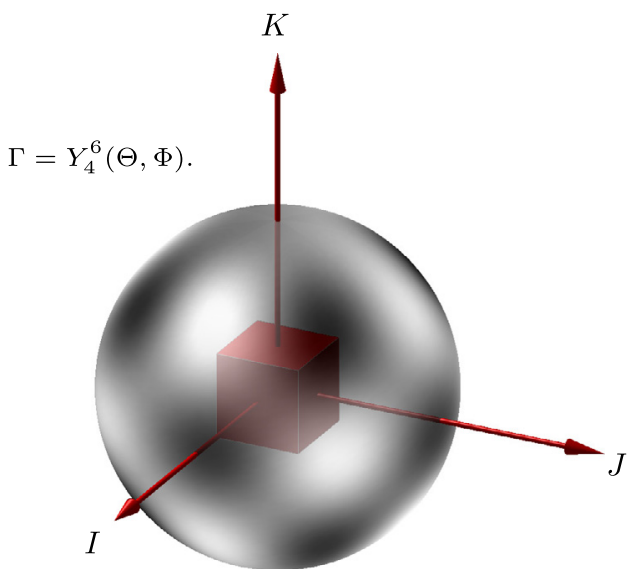

Fig. 13. Spherical harmonic primitive for $l=4$ and $m=6$. 
unconstrained and depends on the mapping between the nodes of the graph and the 3-D data. 3-D Markov-Gibbs random field texture models are used by El-Baz et al. (2008), where co-occurrence of the voxels' gray-level values are modeled by joint Gibbs probability distributions within spherical neighborhoods.

Markov random fields do not require any other prior knowledge than their inherent probability density function. The neighborhood needs to be chosen to be large enough to encompass the largest important structures of the textures.

\subsection{Other approaches}

Other approaches based on 3-D connected components, blobs, watersheds, fractals and Minkowski functionals were used in the literature and are described in the following subsections.

\subsubsection{Connected components, blobs and watersheds}

Analysis of 3-D connected components in binarized images (Martín-Herrero, 2007; Toriwaki and Yoshida, 2009; Sandfort and Ohser, 2009) was used for 3-D medical texture characterization in Paulhac et al. (2009b,a), Paulhac (2009), and Paulhac et al. (2009c). The success of the 3-D connected component approach relies strongly on the critical binarization step that is highly sensitive to illumination differences between datasets. Connected component analysis is also proposed by Ketcham (2005), which extends the blob detection algorithm (Lindeberg, 1993) to 3-D. Uniform components are regrouped based on a given criterion (e.g., Gaussian-smoothed gray levels). Several higher-level measurements are derived from the 3-D blob map such as primitive fitting (analysis of the shapes of blobs) and the contacts between them.

Fetita et al. (2007a,b) and Chang-Chien et al. (2009) propose a hierarchical categorization of the lung structures based on 3-D watersheds. By progressively filling in "basins" defined by 3-D regions with low gray-level values, a hierarchical multi-scale structure of 3-D textures can be obtained from which measures such as the fractal dimension can be derived. This approach was tailored for studying homogeneous regions of lung tissue in MDCT where the gray-level values are univocally expressed in Hounsfield Units (HU) but it may fail in other imaging modalities with varying illumination conditions.

\subsubsection{Minkowski functionals}

Four geometric measures from 3-D Minkowski functionals (Schneider, 1993) are proposed by Boehm et al. (2008) to quantify pulmonary solid textures in binarized MDCT. These measures were also used by van Rikxoort et al. (2011) to detect and quantify emphysema tissue in MDCT. These are the volume, surface area, integral of the mean curvature and Euler-Poincaré components

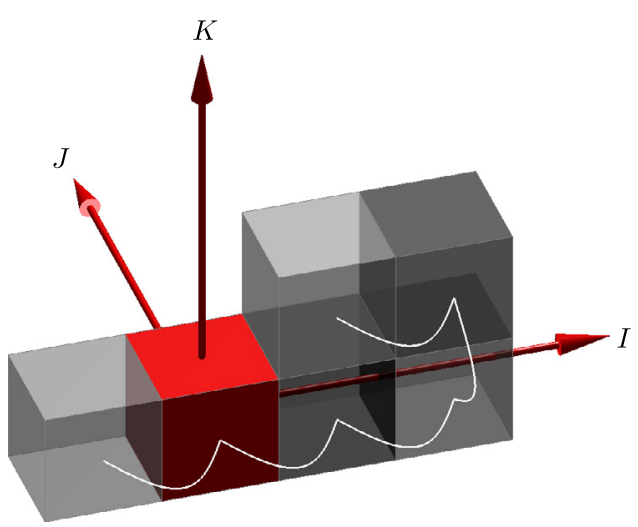

Fig. 14. Markov primitive as a voxel sequence in $\mathbb{Z}^{3}$. (see Boehm et al., 2008 for exact definitions of the measures) computed in binarized MDCT images obtained with thresholds between $-1000 \mathrm{HU}$ and $0 \mathrm{HU}$.

\subsubsection{Fractals}

Fractal dimension from volumetric data is used to characterize 3-D textures by Alberich-Bayarri et al. (2010) and Ollivier et al. (2013) by computing the three-dimensional version of the Minkowski-Bouligand dimension (also called box-counting dimension, Lopes et al., 2011b). It consists of counting the number of boxes of decreasing sizes required to encompass the contour of binarized textures. Similarly to the analysis of 3-D connected components, the success of this method is highly relying on the binarization step. Voxel-based estimation of the stochastic fractal dimension is used in Xu et al. $(2005,2006 b, a)$ to characterize volumetric textural properties in a $5 \times 5 \times 5$ cube around the considered voxel. For all voxel pairs in the cube the average intensity differences versus distances are represented in a log-log plot. The stochastic fractal dimension is then computed as the slope of a linear regression in this plot. This method is scale and rotation invariant but the size of the neighborhood is an important parameter of the algorithm. It may not be appropriate to study textures with prevailing directions because the directional information is discarded.

\subsection{Combination of texture features}

Several approaches for solid 3-D texture analysis used a combination of texture features from various algorithms, relying on the assumption that the heterogeneity of multiple image descriptors allows richer descriptions of the considered textures. Most often texture features are combined with attributes characterizing gray-level distributions (e.g., mean, variance, skewness, kurtosis), because these two information sources are orthogonal.

GLCMs were combined with RLE in Kim and Choi (2009), Georgiadis et al. (2009), Kim et al. (2010), Li et al. (2010), Quellec et al. (2010), Nunzio et al. (2011), Li et al. (2011), Kocinski et al. (2012), and Suoranta et al. (2013) and also with fractal dimension estimation in Huisman et al. (2005), Xu et al. (2005, 2006b,a), Huisman et al. (2007). GLCMs were also used with gradient features in Kovalev et al. (1999, 2001, 2003a,b), Antel et al. (2003), Kovalev and Kruggel (2007), Kovalev and Petrou (2009), Nunzio et al. (2011), Kocinski et al. (2012), and Suoranta et al. (2013) as well as 3-D Gabor filters in Madabhushi et al. (2003) and windowed Fourier in Kontos et al. (2009b).

Venkatraghavan and Ranjan (2012) computed 3-D LBP in the various subbands of 3-D Gabor wavelets. A combination of 2-D and 3-D GLCMs, RLEs, Laws and wavelets is carried out by Basu et al. (2011). van Rikxoort et al. (2011) combined 3-D Minkowski functionals with Laplacian of Gaussian filters.

Most of the combination rules consisted of feature concatenation along with feature selection or reduction (e.g., correlationbased feature selection, principal component analysis). Multiple classifier systems are used in Madabhushi et al. (2003).

\subsection{Quantitative performance analysis and comparison}

The success of a given method depends on the application requirements and it is therefore not meaningful to quantitatively compare the approaches outside of an application context. Some research groups compared the performance and properties of different approaches on a given dataset. Kovalev et al. (1999) compared the properties of gradient orientation histograms and GLCMs on both synthetic data and MRIs of the brain. They found that GLCMs were more robust to noise than the gradient method on the synthetic dataset but less sensitive to subtle changes in microtextures on both datasets. Mariolis et al. (2010b) compared 
the classification accuracy of RLE versus GLCMs for categorizing lung tissue patterns associated with diffuse lung disease in HRCT. Using identical choices of the directions for RLE and GLCMs, they found found no statistical differences between the classification performance. Gao et al. (2010) and Qian et al. (2011) compared the performance of three-dimensional GLCM, LBP, Gabor filters and WT in retrieving similar MR images of the brain. They observed a small increase in retrieval performance for LBP and GLCM when compared to Gabor filters and WT. However, the database used is rather small and the results might not be statistically significant.

Several papers compared the performance of texture analysis algorithms in their 2-D versus 3-D forms. As expected, 2-D texture analysis is most often less discriminative than 3-D, which was observed for various applications and techniques, such as:

- GLCMs, RLE and fractal dimension for the classification of lung tissue types in HRCT in Xu et al. (2005, 2006b).

- GLCMs for the classification of brain tumors in MRI in Mahmoud-Ghoneim et al. (2003) and Allin Christe et al. (2012).

- GLCMs for the classification of breast in contrast-enhanced MRI, where statistical significance was assessed in Chen et al. (2007).

- GMRF for the segmentation of gray matter in MRI in Ranguelova and Quinn (1999).

- LBP for synthetic texture classification in Paulhac et al. (2008).
This demonstrates that 2-D slice-based discrimination of 3-D native texture does not allow fully exploiting the information available in 3-D datasets. An exception was observed with 2-D versus 3-D WTs in Jafari-Khouzani et al. (2004), where the 2-D approach showed a small increase in classification performance of abnormal regions responsible for temporal lobe epilepsy. A separable 3-D WT was used, which did not allow to adequately exploit the 3-D texture information available and may explain the observed results.

\section{Discussion}

In the preceding sections, we have reviewed the current stateof-the-art in 3-D biomedical texture analysis. The papers were categorized in terms of imaging modality used, organ studied and image processing techniques. The increasing number of papers over the past 32 years clearly shows a growing interest in computerized characterization of three-dimensional texture information (see Fig. 5). This is a consequence of increasingly available 3-D data acquisition devices that are reaching high spatial resolutions allowing to capture tissue properties in its natural space.

The analysis of the medical applications in 100 papers in Section 5 shows the diversity of 3-D biomedical textures. The various geometrical properties of the textures are summarized in Table 2 , which defines the multiple challenges of 3-D texture analysis. The need for methods able to characterize structural scales and
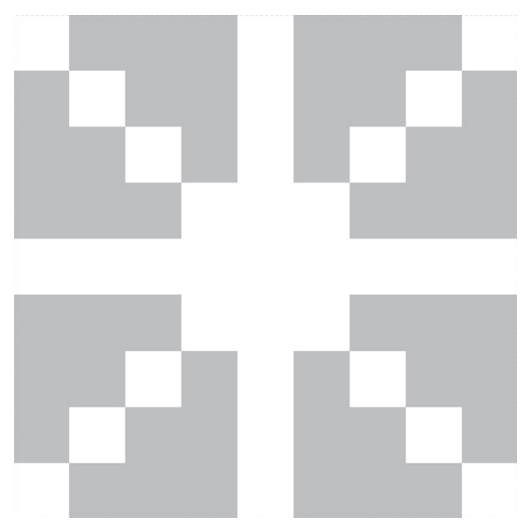

(a) $2-\mathrm{D}$

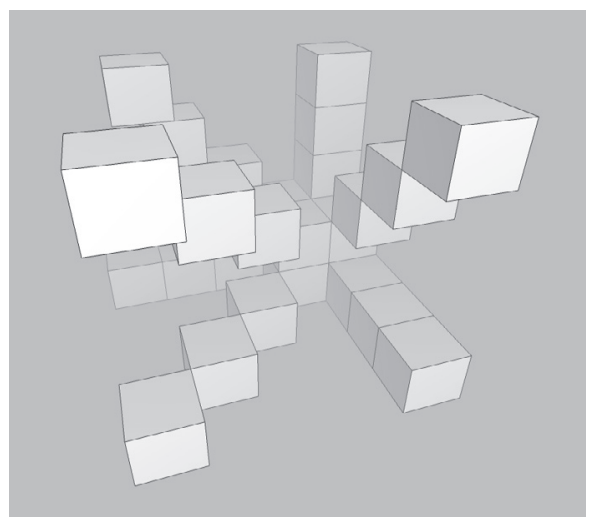

(b) 3-D (one quadrant)

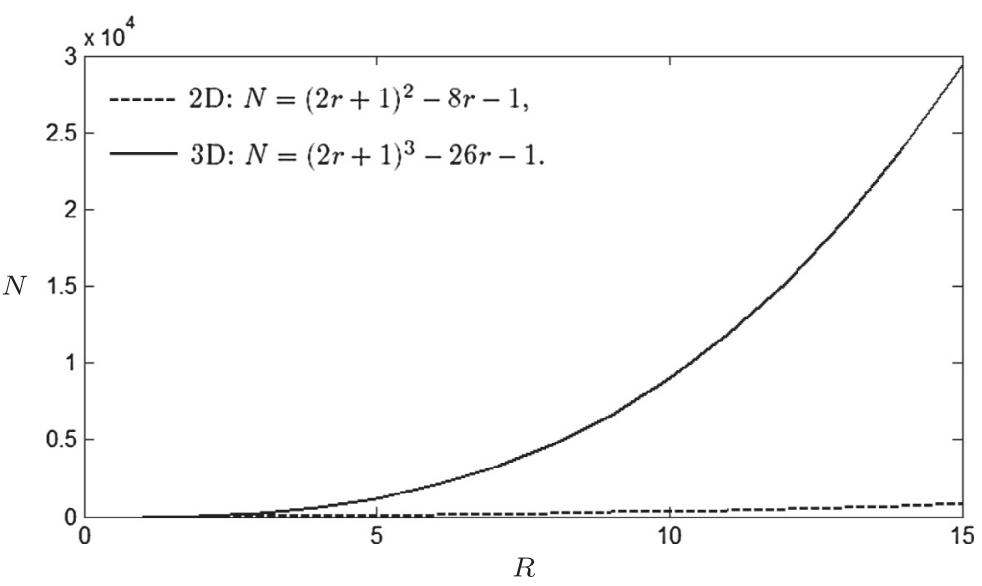

(c) Number $N$ of discarded pixels/voxels according to the neighborhood size $R$.

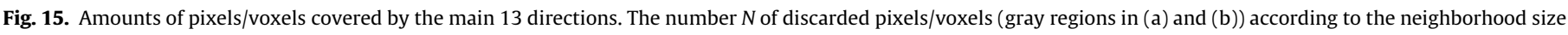
$R$ is shown in (c). 
directions in 3-D Euclidean space with high precision is highlighted. The geometrical properties of the spatial transfer functions of the imaging modalities used are briefly described and compared in Table 1 to understand how biomedical tissue is digitized. It becomes clear that the requirements for successfully characterizing the tissue properties for a given scenario in a given image modality are very diverse and that the computerized approaches must be designed accordingly. The geometric information modeled by the various techniques is analyzed in terms of sets of texture primitives. A qualitative comparison of the ensembles of primitives is proposed in Table 3. The techniques differ in modeling scales and directions of 3-D patterns.

\subsection{Adequation of the texture models}

The size of the structures present in terms of voxels is a very important criterion to consider when choosing a 3-D texture analysis technique. The size of objects $R$ in voxels fixes the number of possible directions as $(2 R+1)^{3}-1$. When small objects are considered (i.e., $R<5$ ), non-convolutional approaches (e.g., GLCMs, RLEs, LBPs) may provide best results. The approximation of the angular domain $(\Theta, \Phi)$ using 13 uniformly distributed directions is acceptable when $R<5$. This is demonstrated in Fig. 15, which depicts the amounts of pixels/voxels that are not covered by the main 13 directions in 2-D/3-D.

When larger objects are considered $R \geqslant 5$, convolutional multiscale approaches are likely to model tissue phenotypes that encompass a series of events occurring at multiple scales and directions. The number of consecutive scales must be chosen to match the spectral properties of the textures. When a dyadic scale progression is used, ${ }^{13}$ the number of scales recommended is $\log _{2}(\bar{R})$, where $\bar{R}$ is the approximate average size of the ROIs (or objects) over the considered database. Using a larger number of scales will increase the influence of objects and textures that are outside the ROIs. Convolutional approaches have also the advantage of providing continuous assessments of the presence of the set of primitives in the signal by providing coefficient maps.

Approaches based on isotropic primitives may provide best results for the characterization of spherical and radial structures (e.g., micronodules, cell nuclei) and when no a priori directions are present in the signal (e.g., diffuse hypo- and hyper-intensities, breast fat). However, the majority of biomedical textures contain directional structures (e.g., vessels, brain gyrifications), and directional approaches are likely to perform best. Anisotropic directional techniques (e.g., separable transforms) can provide sufficient performance when imaging protocols contain inherent anisotropy (e.g., $\Delta z>(\Delta x, \Delta y)$, cylindrical spatial transfer function). The protocols used in clinical routine tend to be increasingly isotropic, and multi-scale convolutional isotropic directional approaches (i.e., non-separable steerable wavelets) are expected to best leverage the wealth of $3-\mathrm{D}$ biomedical texture analysis in the future.

\subsection{Higher-dimensional biomedical texture}

Although biomedical texture exists in dimensions higher than 3 (Nunzio et al., 2011; Foncubierta-Rodríguez et al., 2013c; Depeursinge et al., 2013), no intuitive interactions between the three spatial dimensions and the others can be leveraged easily. The notions of scale and direction do not make sense in non-spatial dimensions. Separate analyses are therefore required, which can be fused together in a second step.

\footnotetext{
${ }^{13}$ A factor of 2 is used between consecutive scales.
}

\subsection{Opportunities}

Several opportunities are available beside the adequate management of the local geometrical properties of the tissue. An important challenge is to reduce the inter-patient and intra-class variations of healthy tissue texture. Future research directions are proposed in this article to tackle this challenge.

All approaches proposed in the literature assume that the texture properties are homogeneous over the whole anatomy, which is not the case in reality. For instance, the bronchovascular structures in the lung parenchyma have very different properties in the mediastinum region when compared to the lung periphery. The breast parenchyma is also highly affected by the presence of lactiferous ducts in the nipple area. Therefore, the creation of texture atlases is required to model local texture properties and to enable accurate comparisons between patients.

Several factors have a strong influence on the texture properties, such as clinical parameters (e.g., age, drugs) and genotypes. Depeursinge et al. (2010) showed the importance of age and hematrocrit level for the classification of lung tissue types associated with interstitial lung disease. In Huo et al. (2002), it was demonstrated that the patient carriers of BRCA1 and BRCA2 mutations tended to have dense breast tissue, and their mammographic patterns tended to be low in contrast, with a coarse texture. A new field called "imaging genomics" studies the link between cellular genomics and tissue-scale imaging (Gevaert et al., 2012; Jaffe, 2012), in which 3-D texture analysis is expected to play a major role. Integrating the overall context of images is therefore essential to better learn the normal appearance of tissue textures.

The combination of the above-mentioned research directions and texture synthesis approaches (e.g., wavelets) provides the exciting opportunity to simulate and visualize the normal ageing process or disease progression for enhanced treatment planning and clinical care management.

\section{Acknowledgments}

This work was supported by the Swiss National Science Foundation (under Grants 205320-141300/1, PBGEP2_142283 and PP00P2-146318), the CIBM, and the EU 7th Framework Program in the context of the Khresmoi project (FP7-257528).

\section{References}

Aguet, F., Jacob, M., Unser, M., 2005. Three-dimensional feature detection using optimal steerable filters. In: IEEE International Conference on Image Processing, pp. II-1158-II-1161.

Aguet, F., Van De Ville, D., Unser, M., 2008. Model-based 2.5-D deconvolution for extended depth of field in brightfield microscopy. IEEE Transactions on Image Processing 17, 1144-1153.

Ahmed, M.N., Farag, A.A., 1996. 3D segmentation and labeling using self-organizing Kohonen network for volumetric measurements on brain CT imaging to quantify TBI recovery. In: Proceedings of the 18th Annual International Conference of the IEEE Engineering in Medicine and Biology Society, 1996. Bridging Disciplines for Biomedicine, pp. 738-739.

Akbari, H., Yang, X., Halig, L.V., Fei, B., 2011. 3D segmentation of prostate ultrasound images using wavelet transform. In: Medical Imaging 2011: Image Processing. SPIE, p. $79622 \mathrm{~K}$.

Alberich-Bayarri, A., Marti-Bonmati, L., Pérez, M.A., Sanz-Requena, R. LermaGarrido, J.J., García-Martí, G., Moratal, D., 2010. Assessment of 2D and 3D fractal dimension measurements of trabecular bone from high-spatial resolution magnetic resonance images at 3 T. Medical Physics 37, 4930-4937.

Alexander, S.K., Azencott, R., Papadakis, M., 2007. Isotropic multiresolution analysis for 3D-textures and applications in cardiovascular imaging. In: Society of PhotoOptical Instrumentation Engineers (SPIE) Conference Series, pp. 67011S1$67011 \mathrm{~S} 12$.

Allin Christe, S., Vasantha Kumari, B., Kandaswamy, A., 2012. Experimental study for 3D statistical property based intracranial brain tumor classification. Journal of Scientific and Industrial Research 71, 36-44.

Andriole, K.P., Wolfe, J.M., Khorasani, R., 2011. Optimizing analysis, visualization and navigation of large image data sets: one 5000-section CT scan can ruin your whole day. Radiology 259, 346-362. 
Antel, S.B., Collins, D.L., Bernasconi, N., Andermann, F., Shinghal, R., Kearney, R.E. Arnold, D.L., Bernasconi, A., 2003. Automated detection of focal cortical dysplasia lesions using computational models of their MRI characteristics and texture analysis. NeuroImage 19, 1748-1759.

Barra, V., Boire, J.Y., 2000. Tissue segmentation on MR images of the brain by possibilistic clustering on a 3D wavelet representation. Journal of Magnetic Resonance Imaging 11, 167-278.

Basu, S., Hall, L.O., Goldgof, D.B., Gu, Y., Kumar, V., Choi, J., Gillies, R.J., Gatenby, R.A., 2011. Developing a classifier model for lung tumors in CT-scan images. In: IEEE International Conference on Systems, Man, and Cybernetics (SMC), pp. 13061312.

Beichel, R., Mitchell, S., Sorantin, E., Leberl, F., Goshtasby, A., Sonka, M., 2001. Shapeand appearance-based segmentation of volumetric medical images. In: International Conference on Image Processing (ICIP 2001), vol. 2, pp. 589-592.

Bennett, R., 1965. Representation and Analysis of Signals Part Xxi. The Intrinsic Dimensionality of Signal Collections. Defense Technical Information Center.

Bhalerao, A., Reyes-Aldasoro, C., 2003. Volumetric texture description and discriminant feature selection for MRI. In: Moreno-Díaz, R., Pichler, F. (Eds.), Lecture Notes in Computer Science (LNCS), Computer Aided Systems Theory EUROCAST 2003, vol. 2809. Springer, Berlin/Heidelberg, pp. 573-584.

Bigun, J., 1994. Speed, frequency, and orientation tuned 3-D Gabor filter banks and their design. In: Proceedings of the 12th IAPR International Conference on Pattern Recognition - Conference C: Signal Processing, pp. 184-187.

Blakemore, C., Campbell, F.W., 1969. On the existence of neurones in the human visual system selectively sensitive to the orientation and size of retinal images. The Journal of Physiology 203, 237-260.

Blot, L., Zwiggelaar, R., 2002. Synthesis and Analysis of Solid Texture: Application in Medical Imaging.

Boehm, H., Fink, C., Attenberger, U., Becker, C., Behr, J., Reiser, M., 2008. Automated classification of normal and pathologic pulmonary tissue by topological texture features extracted from multi-detector CT in 3D. European Radiology 18, 2745 2755.

Bouthemy, P., Fablet, R., 1998. Motion characterization from tempora cooccurrences of local motion-based measures for video indexing. In: Fourteenth International Conference on Pattern Recognition, pp. 905-908.

Bradley, W.G., Brant-Zawadzki, M., Cambray-Forker, J., 2001. MRI of the Brain. Lippincott Williams \& Wilkins.

Burner, A., Donner, R., Mayerhoefer, M., Holzer, M., Kainberger, F., Langs, G., 2012 Texture bags: Anomaly retrieval in medical images based on local 3D-texture similarity. In: Greenspan, H., Müller, H., Syeda-Mahmood, T. (Eds.), Medical Content-based Retrieval for Clinical Decision Support, Lecture Notes in Computer Sciences (LNCS), pp. 116-127.

Cai, W., Liu, S., Wen, L., Eberl, S., Fulham, M.J., Feng, D., 2010. 3D neurological image retrieval with localized pathology-centric CMRGlc patterns. In: 17th IEEE International Conference on Image Processing, pp. 3201-3204.

Candès, E.J., Donoho, D.L., 2000. Curvelets - a surprisingly effective nonadaptive representation for objects with edges. In: Curves and Surface Fitting. Vanderbilt University Press, Nashville, pp. 105-120.

Castellano, G., Bonilha, L., Li, L.M., Cendes, F., 2004. Texture analysis of medical images. Clinical Radiology 59, 1061-1069.

Chan, H.P., Wei, J., Zhang, Y., Helvie, M.A., Moore, R.H., Sahiner, B., Hadjiiski, L. Kopans, D.B., 2008. Computer-aided detection of masses in digital tomosynthesis mammography: comparison of three approaches. Medical Physics 35, 4087-4095.

Chang-Chien, K.C., Fetita, C., Brillet, P.Y., Prêteux, F., Chang, R.F., 2009. Detection and classification of interstitial lung diseases and emphysema using a joint morphological-fuzzy approach. In: Society of Photo-Optical Instrumentation Engineers (SPIE) Conference Series. SPIE, p. 726031.

Chen, X., Murphy, R.F., 2004. Robust classification of subcellular location patterns in high resolution 3D fluorescence microscope images. In: 26th Annual International Conference of the IEEE Engineering in Medicine and Biology Society, pp. 1632-1635.

Chen, W., Giger, M.L., Li, H., Bick, U., Newstead, G.M., 2007. Volumetric texture analysis of breast lesions on contrast-enhanced magnetic resonance images. Magnetic Resonance in Medicine 58, 562-571.

Chenouard, N., Unser, M., 2012. 3D steerable wavelets in practice. IEEE Transactions on Image Processing 21, 4522-4533.

Chomat, O., Crowley, J.L., 1999. Probabilistic recognition of activity using loca appearance. In: Conference on Computer Vision and Pattern Recognition (CCVP 1999), pp. 104-109.

Cula, O.G., Dana, K.J., 2004. 3D texture recognition using bidirectional feature histograms. International Journal of Computer Vision 59, 33-60.

Dana, K.J., Nayar, S.K., 1999. Correlation model for 3Dtexture. In: Proceedings of the Seventh IEEE International Conference on Computer Vision, pp. 1061-1066.

Dana, K.J., van Ginneken, B., Nayar, S.K., Koenderink, J.J., 1999. Reflectance and texture of real-world surfaces. ACM Transactions on Graphics 18, 1-34.

Danciu, M., Gordan, M., Florea, C., Vlaicu, A., 2012. 3D DCT supervised segmentation applied on liver volumes. In: 35th International Conference on Telecommunications and Signal Processing. IEEE, pp. 779-783.

Depeursinge, A., Müller, H., 2011. Medical visual information retrieval based on multi-dimensional texture modeling. In: Proceedings of the 2nd European Future Technologies Conference and Exhibition 2011 (FET 11), pp. 127-129.

Depeursinge, A., Sage, D., Hidki, A., Platon, A., Poletti, P.A., Unser, M., Müller, H., 2007. Lung tissue classification using Wavelet frames. In: 29th Annual International Conference of the IEEE Engineering in Medicine and Biology Society, 2007. EMBS 2007. IEEE Computer Society, Lyon, France, pp. 6259-6262.
Depeursinge, A., Racoceanu, D., Iavindrasana, J., Cohen, G., Platon, A., Poletti, P.A., Müller, H., 2010. Fusing visual and clinical information for lung tissue classification in high-resolution computed tomography. Artificial Intelligence in Medicine 50, 13-21.

Depeursinge, A., Foncubierta-Rodríguez, A., Van De Ville, D., Müller, H., 2011a. Lung texture classification using locally-oriented Riesz components. In: Fichtinger, G., Martel, A., Peters, T. (Eds.), Medical Image Computing and Computer Assisted Intervention - MICCAI 2011. Springer, Berlin/Heidelberg, pp. 231-238.

Depeursinge, A., Zrimec, T., Busayarat, S., Müller, H., 2011b. 3D lung image retrieval using localized features. In: Medical Imaging 2011: Computer-Aided Diagnosis. SPIE, p. 79632E.

Depeursinge, A., Foncubierta-Rodríguez, A., Van De Ville, D., Müller, H., 2012. Multiscale lung texture signature learning using the Riesz transform. In: Medical Image Computing and Computer-Assisted Intervention MICCAI 2012. Springer, Berlin/Heidelberg, pp. 517-524.

Depeursinge, A., Foncubierta-Rodríguez, A., Vargas, A., Van De Ville, D., Platon, A. Poletti, P.A., Müller, H., 2013. Rotation-covariant texture analysis of 4D dualenergy $\mathrm{CT}$ as an indicator of local pulmonary perfusion. In: IEEE 10th International Symposium on Biomedical Imaging. IEEE, pp. 149-152.

Doi, K., 2007. Computer-aided diagnosis in medical imaging: historical review, current status and future potential. Computerized Medical Imaging and Graphics 31, 198-211.

Du, T., Puah, W., Wasser, M., 2011. Cell cycle phase classification in 3D in vivo microscopy of drosophila embryogenesis. BMC Bioinformatics 12, S18.

Duncan, J.S., Ayache, N., 2000. Medical image analysis: progress over two decades and the challenges ahead. IEEE Transactions on Pattern Analysis and Machine Intelligence 22, 85-106.

El-Baz, A., Casanova, M., Gimel'farb, G., Mott, M., Switala, A., Vanbogaert, E., McCracken, R., 2008. Dyslexia diagnostics by 3Dtexture analysis of cerebral white matter gyrifications. In: 19th International Conference on Pattern Recognition, pp. 1-4.

Fehr, J., 2007. Rotational invariant uniform local binary patterns for full 3D volume texture analysis. In: Finnish Signal Processing Symposium (FINSIG), 2007. Oulu, Finland.

Fehr, J., Burkhardt, H., 2006. Phase based 3D texture features, in: Franke, K., Müller, K.R., Nickolay, B., Schäfer, R. (Eds.), Pattern Recognition, Lecture Notes in Computer Science (LNCS), vol. 4174. Springer, Berlin/Heidelberg, pp. 263-272.

Fehr, J., Burkhardt, H., 2008. 3D rotation invariant local binary patterns. In: 19th International Conference on Pattern Recognition, 2008, pp. 1-4.

Fetita, C., Chang-Chien, K.C., Brillet, P.Y., Prêteux, F., Grenier, P., 2007a. Diffuse parenchymal lung diseases: 3D automated detection in MDCT. In: LNCS S. (Ed.), Proceedings of MICCAI 2007, pp. 825-833.

Fetita, C., Chien, K.C.C., Brillet, P.Y., Prêteux, F., 2007b. Automated diagnosis of interstitial lung diseases and emphysema in MDCT imaging. In: Mathematics of Data/Image Pattern Recognition, Compression, Coding, and Encryption X, with Applications. SPIE, p. 67000G.

Filip, J., Haindl, M., 2009. Bidirectional texture function modeling: a state of the art survey. IEEE Transactions on Pattern Analysis and Machine Intelligence 31, $1921-1940$

Foncubierta-Rodríguez, A., Depeursinge, A., Gui, L., Müller, H., 2012. Three dimensional multi-scale visual words for texture-based cerebellum segmentation. In: Medical Imaging 2012: Image Processing. SPIE, p. $83142 Z$.

Foncubierta-Rodríguez, A., Müller, H., Depeursinge, A., 2013a. Retrieval of highdimensional visual data: current state, trends and challenges ahead. Multimedia Tools and Applications, 1-29.

Foncubierta-Rodríguez, A., Poletti, P.A., Platon, A., Vargas, A., Müller, H., Depeursinge, A., 2013b. Texture quantification in 4D dual energy CT for pulmonary embolism diagnosis. In: MICCAI workshop MCBR-CDS 2012, pp. 45-56.

Foncubierta-Rodríguez, A., Jiménez del Toro, O.A., Platon, A., Poletti, P.A., Müller, H., Depeursinge, A., 2013c. Benefits of texture analysis of dual energy CT for computer-aided pulmonary embolism detection. In: The 35th Annual International Conference of the IEEE Engineering in Medicine and Biology Conference (EMBC 2013).

Forkert, N.D., Schmidt-Richberg, A., Fiehler, J., Illies, T., Möller, D., Handels, H., Säring, D., 2011. Fuzzy-based vascular structure enhancement in time-of-flight MRA images for improved segmentation. Methods of Information in Medicine $50,74-83$.

François, R., Fablet, R., Barillot, C., 2003. Robust statistical registration of 3D ultrasound images using texture information. In: Proceedings of the International Conference on Image Processing, 2003, ICIP 2003, pp. 581-584.

Freeman, W.T., Adelson, E.H., 1991. The design and use of steerable filters. IEEE Transactions on Pattern Analysis and Machine Intelligence 13, 891-906.

Galloway, M.M., 1975. Texture analysis using gray level run lengths. Computer Graphics and Image Processing 4, 172-179.

Ganeshan, B., Miles, K.A., Young, R.C.D., Chatwin, C.R., 2008. Three-dimensional selective-scale texture analysis of computed tomography pulmonary angiograms. Investigative Radiology 43, 382-394.

Gao, X., Qian, Y., Hui, R., Loomes, M., Comley, R., Barn, B., Chapman, A., Rix, J., 2010. Texture-based 3D image retrieval for medical applications. In: IADIS Multi Conference on Computer Science and Information System (MCCSIS), pp. 101-108.

Georgiadis, P., Cavouras, D., Kalatzis, I., Glotsos, D., Athanasiadis, E., Kostopoulos, S. Sifaki, K., Malamas, M., Nikiforidis, G., Solomou, E., 2009. Enhancing the discrimination accuracy between metastases, gliomas and meningiomas on brain MRI by volumetric textural features and ensemble pattern recognition methods. Magnetic Resonance Imaging 27, 120-130. 
Gevaert, O., Xu, J., Hoang, C.D., Leung, A.N., Xu, Y., Quon, A., Rubin, D.L., Napel, S., Plevritis, S.K., 2012. Non-small cell lung cancer: identifying prognostic imaging biomarkers by leveraging public gene expression microarray data - methods and preliminary results. Radiology 264, 387-396.

Goldbach, M., Menhardt, W., Stevens, J., 1991. Multispectral tissue characterization in magnetic resonance imaging using bayesian estimation and markov random fields. In: Proceedings of the Annual Conference on Engineering in Medicine and Biology, pp. 62-63.

Hansell, D.M., Bankier, A.A., MacMahon, H., McLoud, T.C., Müller, N.L., Remy, J., 2008. Fleischner society: glossary of terms for thoracic imaging. Radiology 246, 697-722.

Haralick, R.M., 1979. Statistical and structural approaches to texture. Proceedings of the IEEE 67, 786-804.

Haralick, R.M., Shanmugam, K., Dinstein, I., 1973. Textural features for image classification. IEEE Transactions on Systems, Man and Cybernetics 3, 610-621.

Heimann, T., Meinzer, H.P., 2009. Statistical shape models for 3D medical image segmentation: a review. Medical Image Analysis 13, 543-563.

Hoffman, E.A., Reinhardt, J.M., Sonka, M., Simon, B.A., Guo, J., Saba, O., Chon, D., Samrah, S., Shikata, H., Tschirren, J., Palagyi, K., Beck, K.C., McLennan, G., 2003. Characterization of the interstitial lung disease via density-based and texturebased analysis of computed tomography images of lung structure and function. Academic Radiology 10, 1104-1118.

Huisman, A., Ploeger, L.S., Dullens, H.F.J., Poulin, N., Grizzle, W.E., van Diest, P.J., 2005. Development of 3D chromatin texture analysis using confocal laser scanning microscopy. Analytical Cellular Pathology 27, 335-345.

Huisman, A., Ploeger, L.S., Dullens, H.F.J., Jonges, T.N., Belien, J.A.M., Meijer, G.A. Poulin, N., Grizzle, W.E., van Diest, P.J., 2007. Discrimination between benign and malignant prostate tissue using chromatin texture analysis in 3-D by confocal laser scanning microscopy. The Prostate 67, 248-254.

Huo, Z., Giger, M.L., Olopade, O.I., Wolverton, D.E., Weber, B.L., Metz, C.E., Zhong, W., Cummings, S.A., 2002. Computerized analysis of digitized mammograms of BRCA1 and BRCA2 gene mutation carriers. Radiology 225, 519-526.

Ip, H.H.S., Lam, S.W.C., 1994. Using an octree-based rag in hyperirregular pyramid segmentation of texture volume. In: Proceedings of the IAPR Workshop on Machine Vision Applications, pp. 259-262.

Jacob, M., Unser, M., 2004. Design of steerable filters for feature detection using canny-like criteria. IEEE Transactions on Pattern Analysis and Machine Intelligence 26, 1007-1019.

Jafari-Khouzani, K., Soltanian-Zadeh, H., Elisevich, K., Patel, S., 2004. Comparison of 2D and 3D wavelet features for TLE lateralization. In: Amini, A.A., Manduca, A. (Eds.), Medical Imaging 2004: Physiology, Function, and Structure from Medical Images. SPIE, pp. 593-601.

Jaffe, C.C., 2012. Imaging and genomics: is there a synergy? Radiology 264, 329331.

Jain, R.C., Kasturi, R., Schunck, B.G., 1995. Machine Vision, Computer Science Series. McGraw-Hill Higher Education.

Jain, S., Papadakis, M., Upadhyay, S., Azencott, R., 2012. Rigid-motion-invariant classification of 3-D textures. IEEE Transactions on Image Processing 21, 2449 2463.

Jones, J.P., Palmer, L.A., 1987. An evaluation of the two-dimensional Gabor filter model of simple receptive fields in cat striate cortex. Journal of Neurophysiology 58, 1233-1258.

Julesz, B., 1962. Visual pattern discrimination. IRE Transactions on Information Theory 8, 84-92.

Julesz, B., 1981. Textons, the elements of texture perception, and their interactions. Nature 290, 91-97.

Kafieh, R., Rabbani, H., Abramoff, M.D., Sonka, M., 2013. Intra-retinal layer segmentation of 3D optical coherence tomography using coarse grained diffusion map. Medical Image Analysis 17, 907-928.

Kajiya, J.T., Kay, T.L., 1989. Rendering fur with three dimensional textures. SIGGRAPH Computer Graphics 23, 271-280.

Karabulut, N., Kıroglu, Y., 2008. Relationship of parenchymal and pleural abnormalities with acute pulmonary embolism: CT findings in patients with and without embolism. Diagnostic and Interventional Radiology 14, 189-196.

Ketcham, R.A., 2005. Computational methods for quantitative analysis of threedimensional features in geological specimens. Geosphere 1, 32-41.

Kim, T.Y., Choi, H.K., 2009. Computerized renal cell carcinoma nuclear grading using 3D textural features. In: IEEE International Conference on Communications Workshops, 2009, ICC Workshops 2009, pp. 1-5.

Kim, T.Y., Choi, H.J., Hwang, H., Choi, H.K., 2010. Three-dimensional texture analysis of renal cell carcinoma cell nuclei for computerized automatic grading. Journal of Medical Systems 34, 709-716.

Kocinski, M., Klepaczko, A., Materka, A., Chekenya, M., Lundervold, A., 2012. 3D image texture analysis of simulated and real-world vascular trees. Computer Methods and Programs in Biomedicine 107, 140-154.

Kontos, D., Bakic, P.R., Carton, A.K., Troxel, A.B., Conant, E.F., Maidment, A.D.A., 2009a. Parenchymal texture analysis in digital breast tomosynthesis for breast cancer risk estimation: a preliminary study. Academic Radiology 16, 283-298.

Kontos, D., Berger, R., Bakic, P.R., Maidment, A.D.A., 2009b. Breast tissue classification in digital breast tomosynthesis images using texture features: a feasibility study. In: Karssemeijer, N., Giger, M.L. (Eds.), Medical Imaging 2009: Computer-Aided Diagnosis. SPIE, p. 726024.

Korfiatis, P.D., Karahaliou, A.N., Kazantzi, A.D., Kalogeropoulou, C., Costaridou, L.I., 2008. Towards quantification of interstitial pneumonia patterns in lung multidetector CT. In: 8th IEEE International Conference on BioInformatics and BioEngineering, 2008, BIBE 2008, pp. 1-5.
Korfiatis, P.D., Karahaliou, A.N., Costaridou, L.I., 2009. Automated vessel tree segmentation: challenges in computer aided quantification of diffuse parenchyma lung diseases. In: 9th International Conference on Information Technology and Applications in Biomedicine, 2009, ITAB 2009, pp. 1-4.

Korfiatis, P.D., Karahaliou, A.N., Kazantzi, A.D., Kalogeropoulou, C., Costaridou, L.I., 2010. Texture-based identification and characterization of interstitial pneumonia patterns in lung multidetector CT. IEEE Transactions on Information Technology in Biomedicine 14, 675-680.

Korfiatis, P.D., Kalogeropoulou, C., Karahaliou, A.N., Kazantzi, A.D., Costaridou, L.I., 2011. Vessel tree segmentation in presence of interstitial lung disease in MDCT. IEEE Transactions on Information Technology in Biomedicine 15, 214-220.

Kovalev, V.A., Kruggel, F., 2007. Texture anisotropy of the brain's white matter as revealed by anatomical MRI. IEEE Transactions on Medical Imaging 26, 678-685.

Kovalev, V.A., Petrou, M., 1996. Multidimensional co-occurrence matrices for object recognition and matching. Graphical Models in Image Processing 58, 187-197.

Kovalev, V.A., Petrou, M., 2000. Texture analysis in three dimensions as a cue to medical diagnosis. In: Bankman, I.N. (Ed.), Handbook of Medical Imaging. Academic Press, San Diego, pp. 231-247.

Kovalev, V.A., Petrou, M., 2009. Texture analysis in 3D for tissue characterization. In: Bankman, I.N. (Ed.), Handbook of Medical Image Processing and Analysis, second ed. Academic Press, Burlington, pp. 279-292.

Kovalev, V.A., Petrou, M., Bondar, Y.S., 1999. Texture anisotropy in 3-D images. IEEE Transactions on Image Processing 8, 346-360.

Kovalev, V.A., Kruggel, F., Gertz, H.J., von Cramon, D.Y., 2001. Three-dimensiona texture analysis of MRI brain datasets. IEEE Transactions on Medical Imaging 20, 424-433.

Kovalev, V.A., Kruggel, F., von Cramon, D.Y., 2003a. Gender and age effects in structural brain asymmetry as measured by MRI texture analysis. Neurolmage 19, 895-905.

Kovalev, V.A., Petrou, M., Suckling, J., 2003b. Detection of structural differences between the brains of schizophrenic patients and controls. Psychiatry Research: Neuroimaging 124, 177-189.

Kurani, A.S., Xu, D.H., Furst, J., Raicu, D.S., 2004. Co-occurrence matrices for volumetric data. In: The 7th IASTED International Conference on Computer Graphics and Imaging - CGIM 2004. Kauai, Hawaii, USA.

Lang, Z., Scarberry, R.E., Zhang, Z., Shao, W., Sun, X., 1991. A texture-based direct 3D segmentation system for confocal scanning fluorescence microscopic images. In: Twenty-Third Southeastern Symposium on System Theory, pp. 472-476.

Lee, M., Strzelecki, M., 2011. Analysis of three-dimensional magnetic resonance human liver images. IETE Journal of Research 57, 237-245.

Li, X., Xia, H., Zhou, Z., Tong, L., 2010. 3D texture analysis of hippocampus based on MR images in patients with alzheimer disease and mild cognitive impairment. In: 3rd International Conference on Biomedical Engineering and Informatics (BMEI), pp. 1-4.

Li, X., Tong, L.Z., Zhou, X.X., Wang, X., 2011. Classification of 3D texture features based on MR image in discrimination of alzheimer disease and mild cognitive impairment from normal controls. Chinese Journal of Medical Imaging Technology 27, 1047-1051.

Lindeberg, T., 1993. Scale-Space Theory in Computer Vision. Kluwer International Series in Engineering and Computer Science. Kluwer Academic.

Lin, H.C., Wang, L.L., Yang, S.N., 1999. Regular-texture image retrieval based on texture-primitive extraction. Image and Vision Computing 17, 51-63.

Liu, S., Cai, W., Wen, L., Eberl, S., Fulham, M.J., Feng, D., 2011a. Localized functional neuroimaging retrieval using 3D discrete curvelet transform. In: IEEE International Symposium on Biomedical Imaging: From Nano to Macro, pp. 1877-1880

Liu, S., Cai, W., Wen, L., Feng, D.D., 2011b. Volumetric congruent local binary patterns for 3D neurological image retrieval. In: The 26th International Conference on Image and Vision Computing New Zealand (IVCNZ2011), pp. 272-276.

Lopes, R., Ayache, A., Makni, N., Puech, P., Villers, A., Mordon, S., Betrouni, N., 2011a Prostate cancer characterization on MR images using fractal features. Medical Physics 38, 83-95.

Lopes, R., Dubois, P., Bhouri, I., Bedoui, M.H., Maouche, S., Betrouni, N., 2011b. Local fractal and multifractal features for volumic texture characterization. Pattern Recognition 44, 1690-1697.

Luche, C.A.D., Denis, F., Baskurt, A., 2004. 3D steerable pyramid based on conic filters. In: Wavelet Applications in Industrial Processing. SPIE, pp. 260-268.

Lu, X., Jain, A.K., Colbry, D., 2006. Matching 2.5D face scans to 3D models. IEEE Transactions on Pattern Analysis and Machine Intelligence 28, 31-43.

Lu, H., Wang, W., Liao, Q., Zhang, G., Zhou, Z., 2012. Medical image retrieval system using multiple features from 3D ROIs. In: Medical Imaging 2012: Advanced PACS-based Imaging Informatics and Therapeutic Applications. SPIE, pp. 83190E-83190E-8.

Madabhushi, A., Feldman, M.D., Metaxas, D.N., Chute, D., Tomaszeweski, J.E., 2003. A novel stochastic combination of $3 \mathrm{D}$ texture features for automated segmentation of prostatic adenocarcinoma from high resolution MRI. In: 6th International Conference on Medical Image Computing and Computer-Assisted Intervention - MICCAI 2003, pp. 581-591.

Maffei, L., Fiorentini, A., 1973. The visual cortex as a spatial frequency analyser Vision Research 13, 1255-1267.

Mahmoud-Ghoneim, D., Toussaint, G., Constans, J.M., de Certaines, J.D., 2003. Three dimensional texture analysis in MRI: a preliminary evaluation in gliomas. Magnetic Resonance Imaging 21, 983-987.

Mallat, S.G., 1999. A Wavelet Tour of Signal Processing, second ed. Academic Press, San Diego. 
Mariolis, I., Korfiatis, P.D., Costaridou, L.I., Kalogeropoulou, C., Daoussis, D., Petsas, T. 2010a. Investigation of 3D textural features' discriminating ability in diffuse lung disease quantification in MDCT. In: IEEE International Conference on Imaging Systems and Techniques, IST 2010, pp. 135-138.

Mariolis, I., Korfiatis, P.D., Kalogeropoulou, C., Daoussis, D., Petsas, T., Costaridou, L.I., 2010b. Computer aided diagnosis of diffuse lung disease in multi-detector CT selecting 3D texture features. In: Magjarevic, R., Bamidis, P.D., Pallikarakis, N (Eds.), XII Mediterranean Conference on Medical and Biological Engineering and Computing 2010, vol. 29. Springer, Berlin Heidelberg, pp. 208-211.

Marr, D., Hildreth, E., 1980. Theory of edge detection. Proceedings Royal Society of London 207, 187-217.

Martín-Herrero, J., 2007. Hybrid object labelling in digital images. Machine Vision and Applications 18, 1-15.

Materka, A., Strzelecki, M., 1998. Texture Analysis Methods - A Review. Technical Report COST B11 report. Technical University of Lodz. Brussels.

McInerney, T., Terzopoulos, D., 1996. Deformable models in medical image analysis: a survey. Medical Image Analysis 1, 91-108.

Metaxas, D.N., 1996. Physics-Based Deformable Models: Applications to Computer Vision, Graphics, and Medical Imaging, 1st ed. Kluwer Academic Publishers, Norwell, MA, USA.

Mishra, A.K., Kim, D., Andayana, I., 2011. Development of three dimensional binary patterns for local bone structure analysis. In: IEEE International Conference on Bioinformatics and Biomedicine Workshops (BIBMW), pp. 1006-1008.

Moreno, R., Smedby, O., Borga, M., 2011. Soft classification of trabeculae in trabecular bone. In: IEEE International Symposium on Biomedical Imaging: From Nano to Macro, pp. 1641-1644.

Müller, H., Michoux, N., Bandon, D., Geissbuhler, A., 2004. A review of content-based image retrieval systems in medicine-clinical benefits and future directions. International Journal of Medical Informatics 73, 1-23.

Neyret, F., 1995. A general and multiscale model for volumetric textures. in: Graphics Interface. Toronto, Canada, pp. 83-91.

Nishikawa, R.M., 2007. Current status and future directions of computer-aided diagnosis in mammography. Computerized Medical Imaging and Graphics 31, 224-235.

Nunzio, G.D., Pastore, G., Donativi, M., Castellano, A., Falini, A., 2011. A CAD system for cerebral glioma based on texture features in DT-MR images. Nuclear Instruments and Methods in Physics Research Section A: Accelerators, Spectrometers, Detectors and Associated Equipment 648 (Suppl. 1), 100-102.

Odgaard, A., 1997. Three-dimensional methods for quantification of cancellous bone architecture. Bone 20, 315-328.

Ojala, T., Pietikäinen, M., Mäenpää, T., 2002. Multiresolution gray-scale and rotation invariant texture classification with local binary patterns. IEEE Transactions on Pattern Analysis and Machine Intelligence 24, 971-987.

Ollivier, M., Le Corroller, T., Blanc, G., Parratte, S., Champsaur, P., Chabrand, P. Argenson, J.N., 2013. Radiographic bone texture analysis is correlated with 3D microarchitecture in the femoral head, and improves the estimation of the femoral neck fracture risk when combined with bone mineral density. European Journal of Radiology 82, 1494-1498.

Packard, N.J., Abbey, C.K., Yang, K., Boone, J.M., 2012. Effect of slice thickness on detectability in breast CT using a prewhitened matched filter and simulated mass lesions. Medical Physics 39, 1818-1830.

Paulhac, L., 2009. Outils et méthodes d'analyse d'images 3D texturées: application à la segmentation des images échographiques. Ph.D. thesis. Université François Rabelais - Tours, France.

Paulhac, L., Makris, P., Ramel, J.Y., 2008. Comparison between 2D and 3D local binary pattern methods for characterisation of three-dimensional textures. In Proceedings of the 5th International Conference on Image Analysis and Recognition. Springer-Verlag, Berlin, Heidelberg, pp. 670-679.

Paulhac, L., Makris, P., Gregoire, J.M., Ramel, J.Y., 2009a. Approche multirésolution pour la segmentation de textures dans les images ultrasonores 3D. In: XXIIe colloque GRETSI (traitement du signal et des images). Dijon, France.

Paulhac, L., Makris, P., Gregoire, J.M., Ramel, J.Y., 2009b. Descripteurs de textures pour la segmentation d'images echographiques 3D. In: ORASIS'09 - Congrès des jeunes chercheurs en vision par ordinateur. Trégastel, France.

Paulhac, L., Makris, P., Gregoire, J.M., Ramel, J.Y., 2009c. Human understandable features for segmentation of solid texture. In: Proceedings of the 5th International Symposium on Advances in Visual Computing: Part I. SpringerVerlag, Berlin, Heidelberg, pp. 379-390.

Pawley, J.B., 2006. Handbook of Biological Confocal Microscopy. Springer.

Philips, C., Li, D., Raicu, D., Furst, J., 2008. Directional invariance of co-occurrence matrices within the liver. In: Proceedings of the 2008 International Conference on Biocomputation, Bioinformatics, and Biomedical Technologies. IEEE Computer Society, Washington, DC, USA, pp. 29-34.

Qian, Y., Gao, X., Loomes, M., Comley, R., Barn, B., Hui, R., Tian, Z., 2011. Contentbased retrieval of 3D medical images. In: The Third International Conference on eHealth, Telemedicine, and Social Medicine (eTELEMED 2011), IARIA, pp. $7-12$.

Quellec, G., Lee, K., Dolejsi, M., Garvin, M.K., Abramoff, M.D., Sonka, M., 2010. Threedimensional analysis of retinal layer texture: identification of fluid-filled regions in SD-OCT of the macula. IEEE Transactions on Medical Imaging 29, 1321-1330.

Ranguelova, E., Quinn, A., 1999. Analysis and synthesis of three-dimensiona Gaussian Markov random fields. In: Proceedings of the IEEE International Conference on Image Processing, pp. 430-434.

Reed, T.R., du Buf, J.M.H., 1993. A review of recent texture segmentation and feature extraction techniques. CVGIP 57, 359-372.
Reyes-Aldasoro, C.C., Bhalerao, A., 2003. Volumetric texture description and discriminant feature selection for MRI. In: Taylor, C., Noble, J. (Eds.), Information Processing in Medical Imaging, Lecture Notes in Computer Science LNCS, vol. 2732. Springer, Berlin/Heidelberg, pp. 282-293.

Reyes-Aldasoro, C.C., Bhalerao, A., 2007. Volumetric texture segmentation by discriminant feature selection and multiresolution classification. IEEE Transactions on Medical Imaging 26, 1-14.

Sandfort, K., Ohser, J., 2009. Labeling of $n$-dimensional images with choosable adjacency of the pixels. Image Analysis \& Stereology 28.

Sato, Y., Nakajima, S., Shiraga, N., Atsumi, H., Yoshida, S., Koller, T., Gerig, G., Kikinis, R., 1998. Three-dimensional multi-scale line filter for segmentation and visualization of curvilinear structures in medical images. Medical Image Analysis 2, 143-168.

Savio, S.J., Harrison, L.C.V., Luukkaala, T., Heinonen, T., Dastidar, P., Soimakallio, S., Eskola, H.J., 2010. Effect of slice thickness on brain magnetic resonance image texture analysis. BioMedical Engineering OnLine 9, 1-14.

Schneider, R., 1993. Convex Bodies: The Brunn-Minkowski Theory. Encyclopedia of Mathematics and Its Applications. Cambridge University Press.

Schödl, A., Szeliski, R., Salesin, D.H., Essa, I., 2000. Video textures. In: SIGGRAPH '00: Proceedings of the 27th Annual Conference on Computer Graphics and Interactive Techniques. ACM Press/Addison-Wesley Publishing Co., New York, NY, USA, pp. 489-498.

Shafer, C.M., Seewaldt, V.L., Lo, J.Y., 2011. Validation of a 3D hidden-Markov model for breast tissue segmentation and density estimation from MR and tomosynthesis images. In: Biomedical Sciences and Engineering Conference (BSEC), pp. 1-4.

Shen, L., Bai, L., 2008. 3D Gabor wavelets for evaluating SPM normalization algorithm. Medical Image Analysis 12, 375-383.

Showalter, C., Clymer, B., Richmond, B., Powell, K., 2006. Three-dimensional texture analysis of cancellous bone cores evaluated at clinical CT resolutions. Osteoporosis International 17, 259-266.

Simel, D., Drummond, R., 2008. The Rational Clinical Examination: Evidence-Based Clinical Diagnosis. McGraw-Hill.

Simoncelli, E.P., Freeman, W.T., 1995. The steerable pyramid: a flexible architecture for multi-scale derivative computation. In: Proceedings of International Conference on Image Processing, 1995, pp. 444-447.

Smith, J.T., Hawkins, R.M., Guthrie, J.A., Wilson, D.J., Arnold, P.M., Boyes, S., Robinson, P.J., 2010. Effect of slice thickness on liver lesion detection and characterisation by multidetector CT. Journal of Medical Imaging and Radiation Oncology 54, 188-193.

Sørensen, L., Shaker, S.B., Bruijne, M.D., 2010. Quantitative analysis of pulmonary emphysema using local binary patterns. IEEE Transactions on Medical Imaging 29, 559-569.

Suoranta, S., Holli-Helenius, K., Koskenkorva, P., Niskanen, E., Könönen, M., Äikiä, M. Eskola, H., Kälviäinen, R., Vanninen, R., 2013. 3D texture analysis reveals imperceptible MRI textural alterations in the thalamus and putamen in progressive myoclonic epilepsy type 1 . PLoS ONE, 8.

Suzuki, M.T., 2007. A volume data classification method based on 3D spherical filters. In: IEEE International Conference on Systems, Man and Cybernetics, pp. $1095-1100$

Suzuki, M.T., Yaginuma, Y., Noritaka, O., 2004a. A HLAC shape descriptor extraction method for 3D solid textures. WSEAS Transactions on Computers 3, 768-773.

Suzuki, M.T., Yoshitomo, Y., Osawa, N., Sugimoto, Y.Y., 2004b. Classification of solid textures using 3D mask patterns. In: IEEE International Conference on Systems, Man and Cybernetics, 2004, pp. 6342-6347.

Suzuki, M.T., Yaginuma, Y., Yamada, T., Shimizu, Y., 2006. A shape feature extraction method based on 3D convolution masks. In: Eighth IEEE International Symposium on Multimedia (ISM'06). IEEE Computer Society, Los Alamitos, CA, USA, pp. 837-844.

Suzuki, M.T., Yaginuma, Y., Kodama, H., 2009. A texture energy measurement technique for 3D volumetric data. In: IEEE International Conference on Systems, Man and Cybernetics, pp. 3779-3785.

Tamura, H., Mori, S., Yamawaki, T., 1978. Textural features corresponding to visual perception. IEEE Transactions on Systems, Man and Cybernetics 8, 460-473.

Tesar, L., Shimizu, A. Smutek, D., Kobatake, H., Nawano, S, 2008. Medical image analysis of 3D CT images based on extension of Haralick texture features. Computerized Medical Imaging and Graphics 32, 513-520.

Toriwaki, J., Yoshida, H., 2009. Fundamentals of Three-Dimensional Digital Image Processing. Springer.

Jiménez del Toro, O.A., Foncubierta-Rodríguez, A., Vargas Gomez, M.I., Müller, H., Depeursinge, A., 2013. Epileptogenic lesion quantification in MRI using contralateral 3d texture comparisons. In: Medical Image Computing and Computer-Assisted Intervention - MICCAI 2013.

Tourassi, G.D., 1999. Journey toward computer-aided diagnosis: role of image texture analysis. Radiology 213, 317-320.

Unser, M., Van De Ville, D., 2010. Wavelet steerability and the higher-order Riesz transform. IEEE Transactions on Image Processing 19, 636-652.

Upadhyay, S., Papadakis, M., Jain, S., Gladish M.D.G., Kakadiaris, I.A., Azencott, R. 2012. Semi-automatic discrimination of normal tissue and liver cancer lesions in contrast enhanced X-ray CT-scans. In: Yoshida, H., Hawkes, D., Vannier, M. (Eds.), Abdominal Imaging. Computational and Clinical Applications, Lecture Notes in Computer Science, vol. 7601. Springer, Berlin Heidelberg, pp. 158-167.

Van Engelen, A., De Bruijne, M., Klein, S., Verhagen, H., Groen, H., Wentzel, J., van der Lugt, A., Niessen, W., 2011. Plaque characterization in ex vivo MRI evaluated by dense 3D correspondence with histology. In: Medical Imaging 2011: ComputerAided Diagnosis. SPIE, pp. 796329-796329-11. 
van Rikxoort, E.M., Goldin, J.G., Galperin-Aizenberg, M., Brown, M.S., 2011. Classification of pulmonary emphysema from chest CT scans using integral geometry descriptors. In: Medical Imaging 2011: Computer-Aided Diagnosis. SPIE, pp. 796310-796310-6.

Venkatraghavan, V., Ranjan, S., 2012. Semantic analysis of 3D anatomical medica images for sub-image retrieval. In: Greenspan, H., Müller, H., Syeda-Mahmood, T. (Eds.), Medical Content-Based Retrieval for Clinical Decision Support, Lecture Notes in Computer Sciences (LNCS), pp. 139-151.

Webb, W.R., Müller, N.L., Naidich, D.P., 2001. High-Resolution CT of the Lung. Lippincott Williams \& Wilkins, Philadelphia, USA.

Wilson, R., Spann, M., 1988. Finite prolate spheroidal sequences and their applications II: image feature description and segmentation. IEEE Transactions on Pattern Analysis and Machine Intelligence 10, 193-203.

Xu, D.H., Kurani, A.S., Furst, J., Raicu, D.S., 2004. Run-length encoding for volumetric texture. In: The 4th IASTED International Conference on Visualization, Imaging, and Image Processing - VIIP 2004. Marbella, Spain.

Xu, Y., Sonka, M., McLennan, G., Guo, J., Hoffman, E.A., 2005. Sensitivity and specificity of 3-D texture analysis of lung parenchyma is better than 2-D for discrimination of lung pathology in stage 0 COPD. In: Amini, A.A., Manduca, A. (Eds.), SPIE Medical Imaging. SPIE, pp. 474-485.

Xu, Y., van Beek, E.J.R., Hwanjo, Y., Guo, J., McLennan, G., Hoffman, E.A., 2006a. Computer-aided classification of interstitial lung diseases via MDCT: 3D adaptive multiple feature method (3D AMFM). Academic Radiology 13, 969978.

Xu, Y., Sonka, M., McLennan, G., Guo, J., Hoffman, E.A., 2006b. MDCT-based 3D texture classification of emphysema and early smoking related lung pathologies. IEEE Transactions on Medical Imaging 25, 464-475.

Yang, X., Schuster, D., Master, V., Nieh, P., Fenster, A., Fei, B., 2011. Automatic 3D segmentation of ultrasound images using atlas registration and statistical texture prior. In: Medical Imaging 2011: Visualization, Image-Guided Procedures, and Modeling. SPIE, p. 796432.

Yeshurun, Y., Carrasco, M., 2000. The locus of attentional effects in texture segmentation. Nature Neuroscience 3, 622-627.

Ying, L., Demanet, L., Candes, E., 2005. 3D discrete curvelet transform. In: Wavelets XI. SPIE, pp. 591413-591413-11.

Yousefi, S., Zahedi, M., Azmi, R., 2011. Automatic tissue classification in multispectral MRIs via an unsupervised model. World Applied Sciences Journal 12, 1048-1056.

Zhan, Y., Shen, D., 2006. Deformable segmentation of 3-D ultrasound prostate images using statistical texture matching method. IEEE Transactions on Medical Imaging 25, 256-272.

Zucker, S.W., Hummel, R.A., 1981. A three-dimensional edge operator. IEEE Transactions on Pattern Analysis and Machine Intelligence 3, 324-331. 